\title{
Nonlinear Stability of Riemann Ellipsoids with Symmetric Configurations
}

\author{
Miguel Rodríguez-Olmos*, M. Esmeralda Sousa-Dias ${ }^{\dagger}$
}

\begin{abstract}
We apply geometric techniques to obtain the necessary and sufficient conditions on the existence and nonlinear stability of self-gravitating Riemann ellipsoids having at least two equal axes.
\end{abstract}

\section{Introduction}

A Riemann ellipsoid is a relative equilibrium for a dynamical model of a rotating self-gravitating fluid mass that remains an ellipsoid at all times, and for which the fluid velocity is a linear function of the coordinates. This model, first studied and formulated by Dirichlet, is nowadays known as Dirichlet's model. The linearity assumption on the allowed motions makes the study of these deformable bodies very attractive since it implies that their dynamics is governed by a system of ordinary differential equations with a finite number of degrees of freedom. These bodies are also known as affine-rigid bodies or pseudo-rigid bodies. Dirichlet's model can be viewed as a first order model for the study of the shape of the Earth, and the study of the stability of its solutions can be used in planetary stability research.

The study of self-gravitating fluid masses has a long history which can be traced back to Newton's times with many contributions from Dirichlet, MacLaurin, Jacobi, Dedekind, Riemann, Liapunov, Poincaré and Cartan, just to name a few. We can distinguish two classical approaches to the study of the stability of Riemann ellipsoids. One initiated independently in the latter part of the nineteenth century by Poincaré [18] and Liapunov [10] who used expansions in spherical and ellipsoidal harmonics to study the stability of MacLaurin ellipsoids and Jacobi ellipsoids, not only under Dirichlet's assumptions but also under more general conditions (allowing perturbations not preserving the ellipsoidal shape). The other approach occurred in the middle of the twentieth century with the works of Chandrasekhar and collaborators who developed the

\footnotetext{
*Ecole Polytechnique Fédérale de Lausanne (EPFL), Section de Mathématiques. CH-1015 Lausanne, Switzerland, miguel.rodriguez@epfl.ch

†Dep. Matemática, Instituto Superior Técnico, Av. Rovisco Pais, 1049-001 Lisboa, Portugal, edias@math.ist.utl.pt
} 
so-called virial method by applying it to the study of the linear stability of Riemann ellipsoids. These works are collected in the book of Chandrasekhar [3] which constitutes a comprehensive survey on the subject and related problems with many historical facts on this model.

In recent times the subject has had the attention of several researchers, in particular in what respects to the application of new formulations and methods $(24,25])$ to study rotating deformable bodies, not only subject to the self-gravitating potential but also for other potentials modelling nuclei (see for instance [23]), or elastic bodies (see [5] and [9]).

Our aim is to use geometric methods not only to obtain a complete characterization of the conditions for the existence of Riemann ellipsoids having configurations with at least two equal axes but also to obtain the complete description of their nonlinear stability. These geometric methods exploit the geometry and symmetries of the problem and its Hamiltonian structure. Some works using the same philosophical approach to Dirichlet's model are available in the literature for studying several aspects of the problem such as in 20] which obtains Riemann's theorem as a consequence of the symmetry alone, or [8] where some results on the formal stability and bifurcations of MacLaurin spheroids are obtained.

We do not address the problem of the stability for Riemann ellipsoids with three distinct axes for which the self-gravitating potential is an elliptic integral. In [6] this has been studied employing numerical analysis techniques.

We view Dirichlet's model as a Hamiltonian system where Hamilton's function, $h$, has the form kinetic plus potential energy and is defined on the cotangent bundle, $T^{*} \mathrm{SL}(3)$, of the set of all $3 \times 3$ matrices of determinant 1 . Furthermore, $h$ is invariant for the action of $G=\mathbb{Z}_{2} \ltimes(\mathrm{SO}(3) \times \mathrm{SO}(3))$ on the phase space. The $\mathrm{SO}(3) \times \mathrm{SO}(3)$ symmetry reflects the existence of two conserved vector quantities (by Noether's theorem): the angular momentum and circulation. The $\mathbb{Z}_{2}$ symmetry reflects the reciprocity theorem of Dedekind: interchanging the angular velocity and vorticity vectors one obtains another (physically different) solution for Dirichlet's model with the same geometric configuration.

It is well known that Noether's conserved quantities are organized as the components of the momentum map. In the case of relative equilibria with configurations having at least two equal axes (symmetric configurations) the corresponding momentum map value can be singular. Recently, one of the authors developed in 21] a method appropriated for the study of nonlinear stability of this kind of relative equilibria. This construction extends the socalled reduced energy-momentum method of Simo, Lewis and Marsden 26] to the case of singular relative equilibria and so we will refer to the method [21] as the singular reduced energy-momentum method. This is the approach we use in this work to study the nonlinear stability of Riemann ellipsoids with symmetric configurations.

The preliminary sections of the paper are organized as follow: in Section 2 we give the geometric formulation of Dirichlet's model, in Section 3 the singular reduced energy-momentum method is briefly reviewed and in Section 4 we compute the augmented potential energy for symmetric configurations, as a 
necessary step towards the stability analysis.

The main results of the paper are in the following two sections. In Section 5 . Theorem 5.1, we give the complete characterization of all the possible Riemann ellipsoids with symmetric configurations. We prove that for Dirichlet's model the only relative equilibria with configurations having at least two equal axes are: the spherical configuration which has zero angular momentum and circulation, the MacLaurin spheroids which are oblate spheroids rotating around the (shortest) symmetry axis and have angular momentum and circulation aligned with it, and the transversal spheroids, which have prolate spheroidal configurations that rotate around an axis, say $\mathbf{n}$, perpendicular to the (longest) symmetry axis and have angular momentum and circulation aligned with $\mathbf{n}$. We also prove that there are no symmetric relative equilibria for which the angular velocity and vorticity are not aligned with a principal axis of the relative equilibrium configuration. That is, there are not symmetric configurations which are not of type $\mathrm{S}$ in Chandrasekhar's terminology.

In section 6 we apply the singular reduced energy-momentum method to the study of the nonlinear stability of the relative equilibria found in the previous section. The main results of this section are theorems 6.1 6.2 and 6.3 giving respectively necessary and sufficient conditions for the nonlinear stability of the spherical equilibrium, MacLaurin spheroids and transversal spheroids.

Our results on the eccentricity range for the nonlinear $G_{\mu}$-stability of the MacLaurin spheroids agree with those already obtained by Liapunov and Poincaré (see Remark 6.1). In the works of these authors there is no reference to the transversal spheroids, however their existence is acknowledged in pag. 143 of Chandrasekhar's book [3. It is not clear for us what are the results obtained by Chandrasekhar with respect to the stability of the transversal spheroids, however his use of the virial method only gives linear stability. To the best of our knowledge, our result on the nonlinear stability of the transversal spheroids is new.

In conclusion, this work presents, from a purely geometric point of view, a self-contained and complete study of the nonlinear stability of all symmetric relative equilibria for Dirichlet's model. At the same time, the richness of the model helps to clarify the applicability of the singular reduced energymomentum method, providing also a methodology for its application to other models like.

Acknowledgements. We would like to thank to Centro de Análise Matemática, Geometria e Sistemas Dinâmicos of the IST, Lisbon, for the portuguese translation of original Riemann's paper [19] made by C.E. Harle. The work of ESD has been supported by the Fundação para a Ciência e a Tecnologia through the Program POCI 2010/FEDER.

\section{Geometric formulation of Dirichlet's model}

Let $(M, \ll \cdot, \gg)$ be a Riemannian manifold (the configuration manifold), $G$ a Lie group that acts by isometries on $M$ (the symmetry group) and $V \in C^{\infty}(M)$ a 
$G$-invariant function (the potential energy). With these ingredients we construct a symmetric Hamiltonian system on $T^{*} M$ (which is a manifold equipped with a natural symplectic structure) as follows: the potential energy $V$ can be lifted to $T^{*} M$ with the pullback of the cotangent bundle projection $\tau: T^{*} M \rightarrow M$. We denote this lifted function also by $V$. The Riemannian metric on $M$ induces an inner product on each cotangent fiber $T_{x}^{*} M, x \in M$. Then the Hamiltonian is defined as

$$
h\left(p_{x}\right)=\frac{1}{2}\left\|p_{x}\right\|^{2}+V(x), \quad p_{x} \in T_{x}^{*} M .
$$

The $G$-action on $M$ induces a cotangent-lifted Hamiltonian action on $T^{*} M$ with associated equivariant momentum map $\mathbf{J}: T^{*} M \rightarrow \mathfrak{g}^{*}$ defined by

$$
\left\langle\mathbf{J}\left(p_{x}\right), \xi\right\rangle=\left\langle p_{x}, \xi_{M}(x)\right\rangle \quad \forall \xi \in \mathfrak{g},
$$

where $\xi_{M}$ is the fundamental vector field on $M$ associated to the generator $\xi$, defined by

$$
\xi_{M}(x)=\left.\frac{d}{d t}\right|_{t=0} e^{t \xi} \cdot x .
$$

This momentum map is $\mathrm{Ad}^{*}$-equivariant in the sense that $\mathbf{J}\left(g \cdot p_{x}\right)=\operatorname{Ad}_{g^{-1}}^{*} \mathbf{J}\left(p_{x}\right)$ for every $p_{x} \in T_{x}^{*} M, g \in G$.

The Hamiltonian $h$ is $G$-invariant for this lifted action (this follows from the invariance of the metric and of $V$ ). Therefore, due to Noether's theorem, the components of $\mathbf{J}$ are conserved quantities for the Hamiltonian dynamics associated to $h$. The quadruple $(M, \ll \cdot, \cdot \gg, G, V)$ is called a symmetric simple mechanical system.

Let $(M, \ll \cdot, \cdot \gg, G, V)$ be a simple mechanical system with symmetry. A relative equilibrium is a point in phase space $p_{x} \in T^{*} M$ such that its Hamiltonian orbit lies inside a group orbit for the cotangent-lifted action. This amounts to the existence of a generator $\xi \in \mathfrak{g}$ such that the evolution of $p_{x}$ is given by $e^{t \xi} \cdot p_{x}$. The element $\xi$ is called a velocity for the relative equilibrium and is defined up to addition of elements in $\mathfrak{g}_{p_{x}}=\operatorname{Lie}\left(G_{p_{x}}\right)$, where $G_{p_{x}}$ is the stabilizer of $p_{x}$ under the cotangent-lifted action. A useful criterion for finding relative equilibria in simple mechanical systems is given by the following theorem:

Theorem 2.1 (Marsden [11). A point $p_{x} \in T^{*} M$ of a symmetric simple mechanical system $(M, \ll \cdot, \cdot \gg, G, V)$ is a relative equilibrium with velocity $\xi \in \mathfrak{g}$ if and only if the following conditions are verified:

1. $p_{x}=\ll \xi_{M}(x), \cdot \gg$.

2. $x$ is a critical point of $V_{\xi}:=V-\frac{1}{2}\langle\xi, \mathbb{I}(\cdot)(\xi)\rangle$,

where $\mathbb{I}: M \times \mathfrak{g} \rightarrow \mathfrak{g}^{*}$ is defined by $\langle\xi, \mathbb{I}(x)(\eta)\rangle=\ll \xi_{M}(x), \eta_{M}(x) \gg$. Moreover, the momentum $\mu=\mathbf{J}\left(p_{x}\right) \in \mathfrak{g}^{*}$ of the relative equilibrium is given by $\mu=\mathbb{I}(x)(\xi)$.

Note that, in virtue of the above theorem, any relative equilibrium is characterized by a configuration-velocity pair $(x, \xi) \in M \times \mathfrak{g}$ satisfying $\mathbf{d} V_{\xi}(x)=0$. The map II is called the locked inertia tensor, while the function $V_{\xi}$ is called the 
augmented potential. We indicate for later use that the kernel of $\mathbb{I}$ is precisely $\mathfrak{g}_{x}$, the Lie algebra of $G_{x}$, the stabilizer of $x$ for the $G$-action on $M$. The knowledge of the pair $(x, \xi)$ allows us to compute the stabilizer of the corresponding relative equilibrium $p_{x}=\ll \xi_{M}(x), \cdot \gg$ with the formula (see [22]):

$$
G_{p_{x}}=\left\{g \in G_{x}: \operatorname{Ad}_{g} \xi-\xi \in \mathfrak{g}_{x}\right\} .
$$

Dirichlet's model is a symmetric simple mechanical system for the motion of a homogenous and incompressible fluid mass of density $\rho$ having as reference configuration the unit ball centered at the origin in $\mathbb{R}^{3}$ and subject to the selfgravitating potential. The only allowed configurations for this model are linear embeddings of the reference ball into $\mathbb{R}^{3}$ preserving volume and orientation. The configuration manifold $M$ for a self-gravitating fluid mass under Dirichlet's conditions is then SL(3), the group of all $3 \times 3$ matrices with determinant equal to 1 , which is equivalent to the space of orientation and volume preserving linear automorphisms of $\mathbb{R}^{3}$. In what follows we review the geometric formulation of Dirichlet's model as a symmetric simple mechanical system on $G L^{+}(3)$, the group of all $3 \times 3$ matrices with positive determinant, with a symmetric holonomic constraint.

The singular value decomposition of any linear automorphism of $\mathbb{R}^{3}$ allows to decompose (non-uniquely) any matrix $F \in \mathrm{GL}^{+}(3)$ as

$$
F=L A R^{T}
$$

where $L, R \in \mathrm{SO}(3)$, and $A$ is a diagonal matrix with positive entries called singular values (the square roots of the eigenvalues of $C=F^{T} F$ ). It follows from this decomposition that the reference unit ball is mapped by $F$ into a solid ellipsoid of equation $\mathbf{X} \cdot C^{-1} \mathbf{X}=1, \mathbf{X} \in \mathbb{R}^{3}$, having principal axes halflengths equal to the entries of $A$. Physically the matrix $L$ describes the rigid rotation of the body in space relative to an inertial frame and $R$ is related to the rigid internal motion of the fluid with respect to a moving frame. Then $A$ is an orientation preserving dilatation of the original reference body into an ellipsoid with principal axes aligned with the eigenvectors of $A$. The condition on the volume preservation of the total embedding corresponds to impose the holonomic constraint $\operatorname{det} F=1$ (or equivalently $\operatorname{det} A=1$ ), which in turn amounts to consider our system as defined on $\mathrm{SL}(3)$.

The tangent space at $F \in \mathrm{GL}^{+}(3)$ is isomorphic to $\mathrm{L}(3)$, the vector space of $3 \times 3$ matrices. We can define a Riemannian metric on $\mathrm{GL}^{+}(3)$ as:

$$
\ll \delta F_{1}, \delta F_{2} \gg=T \operatorname{tr}\left(\delta F_{1}^{T} \delta F_{2}\right)
$$

for $\delta F_{1}, \delta F_{2} \in T_{F} \mathrm{GL}^{+}(3)$, and $T$ is a constant depending on the density of the reference body and other physical parameters of the system. In the case of interest here, the reference body is a homogeneous unit ball of constant density $\rho$, and $T$ in (10) is

$$
T=\frac{4 \pi}{15} \rho .
$$


The symmetry group $G$ of our model is the semi-direct product $G=\mathbb{Z}_{2} \ltimes$ $(\mathrm{SO}(3) \times \mathrm{SO}(3))$, where $\mathbb{Z}_{2}=\{e, \sigma\}$. Several actions of $G$ of interest in this paper are:

(1) The $G$-action on $G$ : If $(\gamma ; g, h),\left(\gamma^{\prime} ; g^{\prime}, h^{\prime}\right) \in \mathbb{Z}_{2} \ltimes(\mathrm{SO}(3) \times \mathrm{SO}(3))$ then

$$
(\gamma ; g, h) \cdot\left(\gamma^{\prime} ; g^{\prime}, h^{\prime}\right)=\left(\gamma \gamma^{\prime} ;(g, h) \cdot\left(\gamma \cdot\left(g^{\prime}, h^{\prime}\right)\right),\right.
$$

where for the nontrivial element $\sigma \in \mathbb{Z}_{2}, \sigma \cdot\left(g^{\prime}, h^{\prime}\right)=\left(h^{\prime}, g^{\prime}\right)$, and $\mathrm{SO}(3) \times$ $\mathrm{SO}(3)$ acts on itself by the direct product of left matrix multiplications.

(2) The adjoint representation of $G$ : We identify the Lie algebra of $S O(3)$, the set $\mathfrak{s o}(3)$ of skew-symmetric $3 \times 3$ matrices, with $\mathbb{R}^{3}$ via the usual isomorphism ${ }^{\wedge}: \mathbb{R}^{3} \rightarrow \mathfrak{s o}(3):$

$$
v=\left(v_{1}, v_{2}, v_{3}\right) \mapsto \widehat{v}=\left[\begin{array}{ccc}
0 & -v_{3} & v_{2} \\
v_{3} & 0 & -v_{1} \\
-v_{2} & v_{1} & 0
\end{array}\right]
$$

This is an isomorphism of Lie algebras, i.e $(\mathfrak{s o}(3),[\cdot, \cdot])$ is isomorphic by (2) to $\left(\mathbb{R}^{3}, \times\right)$, where $[$,$] denotes the commutator of matrices and \times$ denotes the vector product of vectors in $\mathbb{R}^{3}$.

The Lie algebra of $G$ is then $\mathfrak{g}=\mathbb{R}^{3} \oplus \mathbb{R}^{3}$ and the adjoint action is given by

$$
\operatorname{Ad}_{(\gamma ; g, h)}\left(\xi_{L}, \xi_{R}\right)=\gamma \cdot\left(g \cdot \xi_{L}, h \cdot \xi_{R}\right)
$$

where $\sigma \cdot\left(\xi_{L}, \xi_{R}\right)=\left(\xi_{R}, \xi_{L}\right)$ and $g \cdot \xi_{L}$ is the rotation of $\xi_{L}$ by $g$ (and similarly for $\left.\xi_{R}\right)$.

Using the standard inner product in $\mathbb{R}^{3}$ we also identify $\mathfrak{g}^{*}$ with $\mathbb{R}^{3} \oplus \mathbb{R}^{3}$. Under this identification it follows easily that the coadjoint representation has the expression

$$
\operatorname{Ad}_{(\gamma ; g, h)^{-1}}^{*}\left(\mu_{L}, \mu_{R}\right)=\gamma \cdot\left(g \cdot \mu_{L}, h \cdot \mu_{R}\right)
$$

(3) The $G$-action on $\mathrm{GL}^{+}(3)$ :

$$
(e ; L, R) \cdot F=L F R^{T}, \quad(\sigma ; L, R) \cdot F=R F^{T} L^{T},
$$

Note that the $\mathbb{Z}_{2}$ transposition symmetry on SL(3) (first noticed by Dedekind) maps a rigidly rotating configuration without internal motion into one that is stationary in space but with rigid fluid internal motions. That is, for a given ellipsoid there is an adjoint one, obtained by transposition. These adjoint type of ellipsoids are called Dedekind ellipsoids. More generally, the transposition symmetry interchanges external rotations and internal motions for any solution of Dirichlet's model.

Any $G$-invariant function $f$ on $\mathrm{GL}^{+}(3)$ can be written as

$$
f(F)=\tilde{f}\left(I_{1}(F), I_{2}(F), I_{3}(F)\right),
$$


where $\tilde{f}: \mathbb{R}^{3} \rightarrow \mathbb{R}$, and $I_{1}, I_{2}$ and $I_{3}$ are the three principal invariants of a $3 \times 3$ matrix, given by

$$
\begin{aligned}
I_{1}(F) & =\operatorname{tr}(S) \\
I_{2}(F) & =\frac{1}{2}\left(\operatorname{tr}^{2}(S)-\operatorname{tr}\left(S^{2}\right)\right) \\
I_{3}(F) & =\operatorname{det}(S)
\end{aligned}
$$

with $S=F F^{T}$ (also valid interchanging $S$ with $C=F^{T} F$ ). Note that $I_{1}, I_{2}, I_{3}$ are $G$-invariant and that 1 is a regular value of $I_{3}$. Hence we have $\operatorname{SL}(3)=I_{3}^{-1}(1)$ as a $G$-invariant submanifold of $\mathrm{GL}^{+}(3)$. The condition $I_{3}=1$ is the holonomic constraint of the model. Note also that the restriction of a $G$-invariant function $f \in C^{G}\left(\mathrm{GL}^{+}(3)\right)$ to $\mathrm{SL}(3)$ is given by

$$
f(F)=\tilde{f}\left(I_{1}(F), I_{2}(F), 1\right),
$$

for $F \in \mathrm{SL}(3) \subset \mathrm{GL}^{+}(3)$. Therefore any $G$-invariant function on $\mathrm{SL}(3)$ can be written as $h(F)=\widehat{h}\left(I_{1}(F), I_{2}(F)\right)$, with $\widehat{h}: \mathbb{R}^{2} \rightarrow \mathbb{R}$. Any $G$-invariant function on $\mathrm{SL}(3)$ can be then extended invariantly to $\mathrm{GL}^{+}(3)$ by declaring it to be independent of $I_{3}(F)$. From now on we will drop the tildes and hats from the corresponding functions unless there is risk of confusion.

Since $G$ acts on $\mathrm{GL}^{+}(3)$ by isometries with respect to (1), the induced metric on $\mathrm{SL}(3)$ (which we will denote by the same symbol) is also $G$-invariant. For later use we recall that tangent vectors to $\mathrm{SL}(3)$ can be seen as tangent vectors to $\mathrm{GL}^{+}(3)$ satisfying the linearization of the constraint $I_{3}=1$. In other words,

$$
T_{F} \mathrm{SL}(3)=\left\{\delta F \in \mathrm{L}(3): \operatorname{tr}\left(F^{-1} \delta F\right)=0\right\} .
$$

Dirichlet's model is governed by a Hamiltonian function of the form kinetic plus potential energy on the phase space $\mathcal{P}=T^{*} \mathrm{SL}(3)$ given by

$$
h\left(p_{F}\right)=\frac{T}{2}\left\|p_{F}\right\|^{2}+V(F), \quad p_{F} \in T_{F}^{*} \operatorname{SL}(3) .
$$

Here $\left\|p_{F}\right\|$ is the norm of the covector $p_{F}$ (seen as a $3 \times 3$ matrix) relative to the metric on $\mathrm{SL}(3)$. The potential energy $V$ for a self-gravitating body of homogeneous density $\rho$ under Dirichlet's assumptions is given by restricting the function

$$
V(F)=-R \int_{0}^{\infty} \frac{d s}{\Delta(F)}
$$

where $F \in \mathrm{SL}(3), R=\frac{8}{15} \pi^{2} G \rho^{2}, G$ is the gravitational constant and

$$
\Delta(F)=\sqrt{s^{3}+I_{1}(F) s^{2}+I_{2}(F) s+1} .
$$

The quadruple $\left(\mathrm{SL}(3), \ll \cdot, \cdot \gg, \mathbb{Z}_{2} \ltimes(\mathrm{SO}(3) \times \mathrm{SO}(3)), V\right)$ defines a symmetric simple mechanical system on $\mathrm{SL}(3)$. 
The infinitesimal generator for the $G$-action on $M=\mathrm{GL}^{+}(3)$ (and on SL(3)) corresponding to $\xi=\left(\xi_{L}, \xi_{R}\right) \in \mathbb{R}^{3} \times \mathbb{R}^{3}$ is:

$$
\xi_{M}(F)=\left.\frac{d}{d t}\right|_{t=0}\left(\exp t \widehat{\xi_{L}}, \exp t \widehat{\xi_{R}}\right) \cdot F=\widehat{\xi_{L}} F-F \widehat{F} .
$$

The vectors $\xi_{L}$ and $\xi_{R}$ are respectively the angular velocity and vorticity of the fluid motion. We denote the momentum value of $p_{F}$ by $\mathbf{J}\left(p_{F}\right)=(\mathfrak{j}, \mathfrak{c})$. The components $\mathfrak{j}$ and $\mathfrak{c}$ are respectively the angular momentum and circulation of the instantaneous state $p_{F}$ (see for instance [20] or [3]).

A Riemann ellipsoid (a.k.a. an ellipsoidal figure of equilibrium) is a solution of the Hamiltonian system defined by Dirichlet's model with angular velocity, vorticity and principal axes lengths all constant. In our setting, Riemman ellipsoids correspond exactly to relative equilibria of the underlying symmetric simple mechanical system. Therefore, a Riemann ellipsoid is represented by a triple $\left(F, \xi_{L}, \xi_{R}\right)$, where $F \in \mathrm{SL}(3)$ is the configuration matrix and the Lie algebra element $\left(\xi_{L}, \xi_{R}\right) \in \mathfrak{g}$ is the angular velocity-vorticity pair.

\section{The singular reduced energy-momentum method}

In the last years, several are the works studying the stability of relative equilibria of Hamiltonian systems by exploiting their symmetry and the geometric properties of their phase space (see for instance 2, [15, and [1] for a overview). Most of these methods can be used to test the stability of relative equilibria lying in singular level sets of the momentum map, for instance [7] and [14] under the hypotesis of $G_{\mu}$ compact and [13, [16 using topologic properties. This observation is important since, as we will see, the class of symmetric Riemann ellipsoids known as MacLaurin spheroids corresponds precisely to non-trivial relative equilibria for Dirichlet's model having singular momentum values. We refer the reader to [16] for a comparison of the applicability of several existing methods.

The generally adopted notion of stability for relative equilibria of symmetric Hamiltonian systems is that of $G_{\mu}$-stability, introduced in [15] and that we now review in the context of symmetric simple mechanical systems. This notion is closely related to the Liapunov stability of the induced Hamiltonian system on the reduced phase space.

Definition 3.1. Let $(M, \ll \cdot, \cdot \gg, G, V)$ be a symmetric simple mechanical system and $p_{x} \in T^{*} M$ a relative equilibrium with momentum value $\mu=\mathbf{J}\left(p_{x}\right)$. We say that $p_{x}$ is $G_{\mu}$ stable if for every $G_{\mu}$-invariant neighborhood $U \subset T^{*} M$ of the orbit $G_{\mu} \cdot p_{x}$ there exists a neighborhood $O$ of $p_{x}$ such that the Hamiltonian evolution of $O$ lies in $U$ for all time.

In the early 1990's ([26]) a tool known as the reduced energy-momentum method has been developed, providing sufficient conditions for the stability of relative equilibria of a simple mechanical system under the hypothesis that its momentum is a regular value of the momentum map. This method is especially well-suited for simple mechanical systems since in incorporates all of their 
distinguishing characteristics with respect to general Hamiltonian systems. Recently, based on the characterization [17. of the so-called symplectic normal space $N$ for a cotangent-lifted action, the reduced energy-momentum method was generalized in 21] to cover also the case of singular momentum values.

In this section we outline the implementation of this singular reduced energymomentum method following 21. Our setup will be as in Definition 3.1 and Section 2. In particular we will fix a relative equilibrium $p_{x}$ with configurationvelocity pair $(x, \xi)$ and momentum $\mu$. We will also assume that the $G$-action on $M$ is proper and that there exists a $G_{\mu}$-invariant complement to $\mathfrak{g}_{\mu}$ in $\mathfrak{g}$. These last two conditions are always satisfied for any relative equilibrium in Dirichlet's model due to the compactness of $G$.

We start by stating some key observations: First, by equivariance of $\mathbf{J}$ : $T^{*} M \rightarrow \mathfrak{g}^{*}$ and $\tau: T^{*} M \rightarrow M$, one has $G_{p_{x}} \subset G_{x}$ and $G_{p_{x}} \subset G_{\mu}$. In fact, it is not difficult to prove the characterization

$$
G_{p_{x}}=G_{x} \cap G_{\mu}
$$

We remark that the above formula is not valid in general for covectors $p_{x}$ other than relative equilibria.

Second, also by equivariance of $\tau$ together with the Bifurcation Lemma (see [1]), if $\mu$ is a singular momentum value then $\mathfrak{g}_{x} \neq\{0\}$, in which case $\mu \in$ $\left(\mathfrak{g}_{x}\right)^{\circ}$. Third, the properness of the $G$-action implies that $G_{p_{x}}$ is compact. This, together with (9) allows to define the following $G_{p_{x}}$-invariant splittings:

$$
\mathfrak{g}_{\mu}=\mathfrak{g}_{p_{x}} \oplus \mathfrak{p} \quad \text { and } \quad \mathfrak{g}=\mathfrak{g}_{x} \oplus \mathfrak{p} \oplus \mathfrak{t},
$$

which by duality induce the splittings

$$
\mathfrak{g}_{\mu}^{*}=\mathfrak{g}_{p_{x}}^{*} \oplus \mathfrak{p}^{*} \quad \text { and } \quad \mathfrak{g}^{*}=\mathfrak{g}_{x}^{*} \oplus \mathfrak{p}^{*} \oplus \mathfrak{t}^{*} .
$$

Here $\mathfrak{p}$ and $\mathfrak{t}$ must be chosen in such a way that $\mathbb{I}(x)(\mathfrak{p}) \subset \mathfrak{t}^{\circ}$. Note, from the definition of the locked inertia tensor in Theorem 2.1] that $\operatorname{ker} \mathbb{I}(x)=\mathfrak{g}_{x}$, so the restriction

$$
\widehat{\mathbb{I}}_{0}=\left.\mathbb{I}(x)\right|_{\mathfrak{p} \oplus \mathfrak{t}}: \mathfrak{p} \oplus \mathfrak{t} \rightarrow\left(\mathfrak{g}_{x}\right)^{\circ}=\mathfrak{p}^{*} \oplus \mathfrak{t}^{*}
$$

is a $G_{p_{x}}$-equivariant isomorphism. Then the condition on the above splitting is that $\mathfrak{p}$ and $\mathfrak{t}$ must be orthogonal with respect to the inner product on $\mathfrak{p} \oplus \mathfrak{t}$ induced by $\widehat{\mathbb{I}}_{0}$.

We will denote generically the linear projections associated to the splittings (10) and (11) by the letter $\mathbb{P}$ with an appropriate subindex. For instance $\mathbb{P}_{\mathfrak{p}}$ : $\mathfrak{g} \rightarrow \mathfrak{p}$ or $\mathbb{P}_{\mathfrak{t}^{*}}: \mathfrak{g}^{*} \rightarrow \mathfrak{t}^{*}$. It is a consequence of Noether's theorem that $\xi \in \mathfrak{g}_{\mu}$ and so we will denote by $\xi^{\perp}=\mathbb{P}_{\mathfrak{p}}(\xi) \in \mathfrak{p}$ (the orthogonal velocity of the relative equilibrium).

In this work we only need a particular version of the singular reduced energymomentum method. For, consider the following definitions:

(1) Let $\mathbf{S}$ be the linear orthogonal slice for the $G$-action on $M$ at $x$, i.e.

$$
\mathbf{S}=\left(T_{x}(G \cdot x)\right)^{\perp} \in T_{x} M .
$$


(2) Define the subspace $\mathfrak{q}^{\mu} \subset \mathfrak{g}$ as

$$
\mathfrak{q}^{\mu}=\left\{\gamma \in \mathfrak{t}: \mathbb{P}_{\mathfrak{g}_{x}^{*}}\left[\operatorname{ad}_{\gamma}^{*} \mu\right]=0\right\} .
$$

(3) Define the space of internal variations

$$
\Sigma_{\text {int }}=\left\{\gamma_{M}^{a}+a: \gamma^{a} \in \mathfrak{q}^{\mu}, a \in \mathbf{S}, \text { and }\left(\mathbf{D I} \cdot\left(\gamma_{M}^{a}(F)+a\right)\right)\left(\xi^{\perp}\right) \in \mathfrak{p}^{*}\right\} .
$$

(4) For any $v_{1}, v_{2} \in T_{x} M$, the correction term is the bilinear form on $T_{x} M$ defined by

$$
\operatorname{corr}_{\xi}(x)\left(v_{1}, v_{2}\right)=\left\langle\mathbb{P}_{\mathfrak{p}^{*} \oplus \mathfrak{t}^{*}}\left[\left(\mathbf{D} \mathbb{I} \cdot v_{1}\right)(\xi)\right], \widehat{\mathbb{I}}_{0}^{-1}\left(\mathbb{P}_{\mathfrak{p}^{*} \oplus \mathfrak{t}^{*}}\left[\left(\mathbf{D} \mathbb{I} \cdot v_{2}\right)(\xi)\right]\right)\right\rangle .
$$

(5) The Arnold form Ar: $\mathfrak{q}^{\mu} \times \mathfrak{q}^{\mu} \rightarrow \mathbb{R}$ is defined by:

$$
\operatorname{Ar}\left(\gamma_{1}, \gamma_{2}\right)=\left\langle\operatorname{ad}_{\gamma_{1}}^{*} \mu, \widehat{\mathbb{I}}_{0}^{-1}\left(\operatorname{ad}_{\gamma_{2}} \mu\right)+\mathbb{P}_{\mathfrak{p} \oplus \mathfrak{t}}\left[\operatorname{ad}_{\gamma_{2}}\left(\widehat{\mathbb{I}}_{0}^{-1} \mu\right)\right]\right\rangle .
$$

The following theorem (Corollary 6.2 of [21]) is the synthesis of the singular reduced energy-momentum method.

Theorem 3.1. Let $p_{x} \in T^{*} M$ be a relative equilibrium with configurationvelocity pair $(x, \xi) \in M \times \mathfrak{g}$ and momentum $\mu \in \mathfrak{g}^{*}$ such that $\operatorname{dim}(G \cdot F)<\operatorname{dim} M$. Let $\xi^{\perp}=\mathbb{P}_{\mathfrak{p}}(\xi)$ be its orthogonal velocity. If the Arnold form is non-degenerate at $p_{x}$ and $\left.\left(\mathbf{d}_{x}^{2} V_{\xi^{\perp}}+\operatorname{corr}_{\xi^{\perp}}(x)\right)\right|_{\Sigma_{\mathrm{int}}}$ is positive definite, then the relative equilibrium is $G_{\mu}$-stable.

When the Arnold form is non-degenerate it is also shown in 21] that the symplectic matrix of the symplectic normal space at $p_{x}$ has a particularly simple block-diagonal expression. We quote this result which will be essential in the proof of the linear unstability of some Riemann ellipsoids.

Theorem 3.2. If the Arnold form is non-degenerate at the relative equilibrium $p_{x}$ with configuration-velocity pair $(x, \xi)$ and momentum $\mu$, the symplectic normal space at $p_{x}$, is symplectomorphic to $N=\mathfrak{q}^{\mu} \oplus \Sigma_{\mathrm{int}} \oplus \mathbf{S}^{*}$ equipped with the symplectic matrix

$$
\omega_{N}=\left[\begin{array}{ccc}
\mathfrak{q}^{\mu} & \Sigma_{\text {int }} & \mathbf{S}^{*} \\
\Xi & -\Psi & 0 \\
\Psi^{T} & -\left.\mathbf{d} \chi^{\xi^{\perp}}\right|_{\Sigma_{\text {int }}} & \mathbf{1} \\
0 & -\mathbf{1} & 0
\end{array}\right]
$$

where

$$
\Xi\left(\gamma_{1}, \gamma_{2}\right)=-\left\langle\mu, \operatorname{ad}_{\gamma_{1}} \gamma_{2}\right\rangle, \quad \Psi\left(\gamma,\left(\gamma_{M}^{b}(x)+b\right)\right)=\left\langle\mu, \operatorname{ad}_{\gamma} \gamma^{b}\right\rangle
$$

and $\chi^{\xi^{\perp}}$ is the one-form defined by $\chi^{\xi^{\perp}}(X)=\ll \xi_{M}^{\perp}, X \gg$, for all $X \in \mathfrak{X}(M)$. 
Remark 3.1. The $G$-invariance of $V$ and $\ll \cdot, \cdot \gg$ imply the following property. If $(x, \xi)$ is a relative equilibrium, then the orbit $\left(g \cdot x, \operatorname{Ad}_{g} \xi\right)$ for every $g \in G$ consists of relative equilibria with the same stability or unstability properties.

Remark 3.2. The reason why in the previous section we looked at Dirichlet's model as a simple mechanical system holonomicaly constrained is that the unconstrained space $\mathrm{GL}^{+}(3)$ is an open domain of the vector space $\mathrm{L}(3)$, and then the implementation of the reduced energy-momentum method is easier than if one is working directly on $\mathrm{SL}(3)$. In view of the survey of the method, the strategy will be to use the trivial extension of the self-gravitating potential to $\mathrm{GL}^{+}(3)$ and consider its augmented potential with respect to the locked inertia tensor corresponding to the original Riemannian metric on $\mathrm{GL}^{+}(3)$. Then we further augment this augmented potential with the constraint function $I_{3}$ and Lagrange multiplier $\lambda$. Denoting by

$$
V_{\xi_{L}, \xi_{R}}^{\lambda}=V_{\xi_{L}, \xi_{R}}-\lambda \operatorname{det}
$$

the resulting twice augmented potential, we have:

(1) Relative equilibria for Dirichlet's model correspond to triples $\left(F, \xi_{L}, \xi_{R}\right)$ with $F \in \mathrm{GL}^{+}(3),\left(\xi_{L}, \xi_{R}\right) \in \mathbb{R}^{3} \times \mathbb{R}^{3}$ such that the following two equations hold:

$$
\mathbf{d} V_{\xi_{L}, \xi_{R}}^{\lambda}(F)=0, \quad \text { and } \quad \operatorname{det}(F)=1 .
$$

(2) The stability test now follows from the following observation. If we call $\Sigma_{\text {int }}^{\mathrm{GL}^{+}(3)}$ and $\Sigma_{\text {int }}^{\mathrm{SL}(3)}$ the spaces of internal variations for $\mathrm{GL}^{+}(3)$ and $\mathrm{SL}(3)$ associated to the triple $\left(F, \xi_{L}, \xi_{R}\right)$, according to (12), we notice that

$$
\Sigma_{\text {int }}^{\mathrm{SL}(3)}=\Sigma_{\text {int }}^{\mathrm{GL}^{+}(3)} \cap \operatorname{ker} T_{F} I_{3} .
$$

Therefore, according to the general method, and standard Lagrange multiplier theory, to conclude stability it suffices to study the eigenvalues of the bilinear form

$$
\left.\left(\mathbf{d}_{F}^{2} V_{\left(\xi_{L}, \xi_{R}\right)^{\perp}}^{\lambda}+\operatorname{corr}_{\left(\xi_{L}, \xi_{R}\right)^{\perp}}(F)\right)\right|_{\Sigma_{\mathrm{int}}^{\mathrm{SL}(3)}},
$$

where $\Sigma_{\text {int }}^{\mathrm{SL}(3)}$ is seen as a vector subspace of $\Sigma_{\text {int }}^{\mathrm{GL}^{+}(3)}$. From now on we will omit the superindex SL(3) for the space of internal variations.

\section{The augmented self-gravitating potential for symmetric configurations}

In this section we compute the augmented potential

$$
V_{\xi_{L}, \xi_{R}}=V-\frac{1}{2}\left\langle\left(\xi_{L}, \xi_{R}\right), \mathbb{I}(F)\left(\xi_{L}, \xi_{R}\right)\right\rangle
$$


in the unconstrained configuration space $\mathrm{GL}^{+}(3)$ and collect some results for the self-gravitating potential $V$. The potential $V(F)$ at a typical configuration is an elliptic integral except for symmetric configurations (i.e. with at least two equal singular values) for which it can be integrated by elementary functions. The extension to the unconstrained space of the potential $V$ depends on $F \in$ $\mathrm{GL}^{+}(3)$ through the two principal invariants $I_{1}$ and $I_{2}$ defined by (3) and (4) respectively. In the study of the existence and stability of relative equilibria of simple mechanical systems will be necessary to compute the first and second derivatives of $V_{\xi_{L}, \xi_{R}}$ and the results of this section are essential to this end. In the following we will restrict ourselves to diagonal configurations only. There is no loss of generality with this assumption since, according to the singular value decomposition, every matrix $F \in \mathrm{GL}^{+}(3)$ belongs to the $G$-orbit of some diagonal configuration $D$ by some element $(e ; A, B) \in G$. Hence by Remark 3.1 the qualitative properties of a relative equilibrium $\left(D,\left(\xi_{L}, \xi_{R}\right)\right)$ are the same as those of $\left(A D B^{T},\left(A \xi_{L}, B \xi_{R}\right)\right)$.

Let $J(k, r)$, with $k, r \in \mathbb{N}$ be the following family of integrals:

$$
J(k, r):=\int_{0}^{\infty} \frac{s^{r} d s}{\Delta(F)^{k}},
$$

and denote by $V_{i}(i=1,2)$ the partial derivative of $V$ with respect to $I_{i}$ and by $V_{i j}$ the partial derivative of $V_{i}$ with respect to $I_{j}$ for $j=1,2$. Using (6), elementary calculus computations give:

$$
\begin{aligned}
& V=-R J(1,0) \quad V_{1}=\frac{R}{2} J(3,2) \quad V_{2}=\frac{R}{2} J(3,1) \\
& V_{11}=-\frac{3 R}{4} J(5,4) \quad V_{12}=-\frac{3 R}{4} J(5,3) \quad V_{22}=-\frac{3 R}{4} J(5,2) \text {. }
\end{aligned}
$$

Note that the integrals $J(k, r)$ are all positive as well as $V_{1}$ and $V_{2}$.

Next proposition gives the values of $J(k, r)$ in the case of spheroidal (two equal axes) configurations.

Proposition 4.1. Let $F=\operatorname{diag}(a, a, c)$ be a spheroidal configuration with $a^{2} c=$ 1.

(i) The integral $J(k, r)$ for the oblate spheroid $F(a>c)$ and eccentricity $e=\sqrt{1-\left(\frac{c}{a}\right)^{2}}$ is given by

$$
J_{O}(k, r)=\frac{2}{\left(1-e^{2}\right)^{\frac{2(r+1)-3 k}{6}}} \int_{0}^{1} \frac{\left(1-x^{2}\right)^{r} x^{3(k-1)-2 r} d x}{\left(1-e^{2} x^{2}\right)^{\frac{k}{2}}} .
$$

(ii) The integral $J(k, r)$ for the prolate spheroid $F(a<c)$ and eccentricity $e=\sqrt{1-\left(\frac{a}{c}\right)^{2}}$ is given by

$$
J_{P}(k, r)=\frac{2}{\left(1-e^{2}\right)^{\frac{2(r+1)-3 k}{3}}} \int_{0}^{1} \frac{\left(1-x^{2}\right)^{r} x^{3(k-1)-2 r} d x}{\left(1-e^{2} x^{2}\right)^{k}} .
$$


Proof. Note that for the diagonal configuration $F=\operatorname{diag}(a, b, c)$ the value of $\Delta(F)$ in definition (7) is

$$
\Delta(F)=\left[\left(a^{2}+s\right)\left(b^{2}+s\right)\left(c^{2}+s\right)\right]^{1 / 2} .
$$

For (i): making the change of variables $s=a^{2} \tan ^{2} \theta$ we get

$$
J_{O}(k, r)=2 a^{2(r+1)-3 k} \int_{0}^{\pi / 2} \frac{(\sin \theta)^{2 r+1}(\cos \theta)^{3(k-1)-2 r}}{\left(1+\frac{c^{2}-a^{2}}{a^{2}} \cos ^{2} \theta\right)^{k / 2}} d \theta .
$$

Since the eccentricity is $e^{2}=\frac{a^{2}-c^{2}}{a^{2}}$ then $a=\left(1-e^{2}\right)^{-1 / 6}$ and $c=\left(1-e^{2}\right)^{1 / 3}$ because $a^{2} c=1$. Then, from the above expression for $J_{O}(k, r)$ one gets

$$
J_{O}(k, r)=\frac{2}{\left(1-e^{2}\right)^{\frac{2(r+1)-3 k}{6}}} \int_{0}^{\pi / 2} \frac{(\sin \theta)^{2 r+1}(\cos \theta)^{3(k-1)-2 r}}{\left(1-e^{2} \cos ^{2} \theta\right)^{k / 2}} d \theta .
$$

Making $x=\cos \theta$ the result follows.

For (ii): The change of variables $s=c^{2} \tan ^{2} \theta$ gives

$$
J_{P}(k, r)=2 c^{2(r+1)-3 k} \int_{0}^{\pi / 2} \frac{(\sin \theta)^{2 r+1}(\cos \theta)^{3(k-1)-2 r}}{\left(1+\frac{a^{2}-c^{2}}{c^{2}} \cos ^{2} \theta\right)^{k}} d \theta .
$$

The eccentricity $e$ of the prolate spheroid is such that $c^{2}\left(1-e^{2}\right)=a^{2}$. As $a^{2} c=1$ then $a=\left(1-e^{2}\right)^{1 / 6}$ and $c=\left(1-e^{2}\right)^{-1 / 3}$ and the result follows for $x=\cos \theta$.

As stated in the previous section, in order to find critical points of a $G$ invariant function defined in $\mathrm{SL}(3)$ we will work with its extension to $\mathrm{GL}^{+}(3)$ subject to the constraint $\operatorname{det} F=1$. For, since any such function can be written as $f(F)=f\left(I_{1}(F), I_{2}(F)\right)$, in order to compute the critical points we use the augmented function $f^{\lambda}(F)=f\left(I_{1}(F), I_{2}(F)\right)-\lambda \operatorname{det}(F)$ subject to the condition $\operatorname{det}(F)=1$. For the differentiation of $f^{\lambda}$ consider the pairing between vectors $\delta F \in T_{F} S L(3)$ and covectors $B \in T_{F}^{*} \mathrm{SL}(3)$ to be $B \cdot \delta F=\operatorname{tr}\left(B^{T} \delta F\right)$. Then using the chain rule we get that critical points must verify the following set of equations:

$$
\begin{aligned}
\mathbf{d} f^{\lambda}(F) \cdot \delta F & =2 \operatorname{tr}\left[\left(f_{1} F^{T}+f_{2}\left(I_{1} F^{T}-F^{T} F F^{T}\right)-\frac{\lambda}{2} \operatorname{det}(F) F^{-1}\right) \delta F\right](20) \\
& =0 \\
\operatorname{det}(F) & =1
\end{aligned}
$$

since

$$
\begin{aligned}
\mathbf{d} I_{1}(F) \cdot \delta F & =2 \operatorname{tr}\left(F^{T} \delta F\right) \\
\mathbf{d} I_{2}(F) \cdot \delta F & =2\left[I_{1} \operatorname{tr}\left(F^{T} \delta F\right)-\operatorname{tr}\left(F^{T} F F^{T} \delta F\right)\right] \\
\mathbf{d} \operatorname{det}(F) \cdot \delta F & =\operatorname{det}(F) \operatorname{tr}\left(F^{-1} \delta F\right) .
\end{aligned}
$$

(see for instance [4] or [12]).

Next proposition gives the expression for the locked inertia tensor. 
Proposition 4.2. The locked inertia tensor for the $G$-action on $T^{*} \mathrm{GL}^{+}(3)$, at a configuration $F \in \mathrm{GL}^{+}(3)$, is defined by

$\left\langle\left(\widehat{\xi_{L}}, \widehat{\xi_{R}}\right), \mathbb{I}(F)\left(\widehat{\eta_{L}}, \widehat{\eta_{R}}\right)\right\rangle=T \operatorname{tr}\left[{\widehat{\xi_{L}}}^{T} \widehat{\eta_{L}} F F^{T}+{\widehat{\xi_{R}}}^{T} F^{T} F \widehat{\eta_{R}}-{\widehat{\xi_{L}}}^{T} F{\widehat{\eta_{R}}}^{T}-{\widehat{\xi_{R}}}^{T} F^{T} \widehat{\eta_{L}} F\right]$

where $T=\frac{4 \pi}{15} \rho$ and $\widehat{\xi}_{i}, \widehat{\eta}_{i} \in \mathfrak{s o}(3)$ for $i=1,2$.

Under the isomorphism (2), the locked inertia tensor is also equivalent to:

$$
\left\langle\left(\xi_{L}, \xi_{R}\right), \mathbb{I}(F)\left(\eta_{L}, \eta_{R}\right)\right\rangle=T\left[\begin{array}{ll}
\xi_{L} & \xi_{R}
\end{array}\right]\left[\begin{array}{cc}
\mathbf{i}_{S} & -2 \operatorname{det}(F) F^{-T} \\
-2 \operatorname{det}(F) F^{-1} & \mathbf{i}_{C}
\end{array}\right]\left[\begin{array}{l}
\eta_{L} \\
\eta_{R}
\end{array}\right]
$$

where $S=F F^{T}, C=F^{T} F$ and $\mathbf{i}_{A}=\operatorname{tr}(A) \mathbf{I}-A$ (I denotes the identity matrix).

Proof. By the locked inertia tensor definition in Proposition 2.1, the expression (8) for the infinitesimal generators of the $G$-action on $\mathrm{GL}^{+}(3)$ and the definition (11) for the Riemannian metric, we have

$$
\begin{aligned}
\left\langle\left(\widehat{\xi_{L}}, \widehat{\xi_{R}}\right), \mathbb{I}(F)\left(\widehat{\eta_{L}}, \widehat{\eta_{R}}\right)\right\rangle & =\ll\left(\widehat{\xi_{L}}, \widehat{\xi_{R}}\right)_{\mathrm{GL}^{+}(3)}(F),\left(\widehat{\eta_{L}}, \widehat{\eta_{R}}\right)_{\mathrm{GL}^{+}(3)}(F) \gg \\
& =\ll \widehat{\xi_{L}} F-F \widehat{\xi_{R}}, \widehat{\eta_{L}} F-F \widehat{\eta_{R}} \gg \\
& =T \operatorname{tr}\left[\left(\widehat{\xi_{L}} F-F \widehat{\xi_{R}}\right)^{T}\left(\widehat{\eta_{L}} F-F \widehat{\eta_{R}}\right)\right] .
\end{aligned}
$$

Using the fact that $\widehat{\xi}_{i}$ and $\widehat{\eta}_{i}$ are skew-symmetric matrices and the cyclic property of the trace of a matrix, it is straightforward to obtain expression (24).

For the expression (25) we need some standard properties of the isomorphism (2). In particular,

$$
\begin{aligned}
& \operatorname{tr}\left(\widehat{\xi}^{T} \widehat{\eta}\right)=2 \xi \cdot \eta \\
& \operatorname{tr}(\widehat{\xi} L)=\frac{1}{2} \operatorname{tr}\left(\widehat{\xi}\left(L-L^{T}\right)\right) \\
& L \widehat{\xi}+\widehat{\xi} L=\widehat{\mathbf{i}_{L} v} \quad \text { if } L \text { is a symmetric matrix } \\
& \widehat{L \xi}=\operatorname{det}(L) L^{-T} \widehat{\xi} L^{-1} \quad \text { if } L \text { is an invertible matrix }
\end{aligned}
$$

where $\cdot$ denotes the standard inner product on $\mathbb{R}^{3}$. Let us compute some terms of the expression (24) since the other are done similarly

$$
\begin{aligned}
\operatorname{tr}\left({\widehat{\xi_{L}}}^{T} \widehat{\eta_{L}} F F^{T}\right) & =\frac{1}{2} \operatorname{tr}\left[{\widehat{\xi_{L}}}^{T}\left(\widehat{\eta_{L}} F F^{T}+F F^{T} \widehat{\eta_{L}}\right)\right] & & \text { (by (27)) } \\
& =\frac{1}{2} \operatorname{tr}\left({\widehat{\xi_{L}}}^{T} \widehat{\mathbf{i}}_{S} \eta_{L}\right) & & \text { (by (28) }) \\
& =\xi_{L} \cdot \mathbf{i}_{S} \eta_{L} & & \text { (by (26) })
\end{aligned}
$$




$$
\begin{aligned}
\operatorname{tr}\left({\widehat{\xi_{R}}}^{T} F^{T} \widehat{\eta_{L}} F\right) & =\frac{1}{\operatorname{det}\left(F^{-1}\right)} \operatorname{tr}\left[{\widehat{\xi_{R}}}^{T} \widehat{F^{-1} \eta_{L}}\right] & & \text { (by (29)) } \\
& =2 \operatorname{det}(F) \xi_{R} \cdot F^{-1} \eta_{L} & & \text { (by (26) })
\end{aligned}
$$

As a straightforward consequence we can obtain the momentum of a relative equilibrium for Dirichlet's model, that is its angular momentum and circulation.

Corollary 4.1. The momentum for a relative equilibrium with configuration $F$ and velocity-vorticity pair $\left(\xi_{L}, \xi_{R}\right) \in \mathbb{R}^{3} \oplus \mathbb{R}^{3}$ is

$$
\mu=\mathbb{I}(F)\left(\xi_{L}, \xi_{R}\right)=T\left(\mathbf{i}_{S} \xi_{L}-2 \operatorname{det}(F) F^{-T} \xi_{R}, \mathbf{i}_{C} \xi_{R}-2 \operatorname{det}(F) F^{-1} \xi_{L}\right) .
$$

That is, the angular momentum and circulation of a Riemann ellipsoid with configuration by $F$, and angular velocity-vorticity pair $\left(\xi_{L}, \xi_{R}\right)$ are given, respectively, by

$$
\begin{aligned}
\mathfrak{j} / T & =\mathbf{i}_{S} \xi_{L}-2 \operatorname{det}(F) F^{-T} \xi_{R} \\
\mathfrak{c} / T & =\mathbf{i}_{C} \xi_{R}-2 \operatorname{det}(F) F^{-1} \xi_{L}
\end{aligned}
$$

The expression for the twice augmented potential $V_{\xi_{L}, \xi_{R}}^{\lambda}$ follows now easily from Proposition 4.2

$$
\begin{aligned}
V_{\xi_{L}, \xi_{R}}^{\lambda}(F)= & -R \int_{0}^{\infty} \frac{d s}{\Delta(F)} \\
& -T\left(\frac{1}{2} \xi_{L} \cdot \mathbf{i}_{S} \xi_{L}+\frac{1}{2} \xi_{R} \cdot \mathbf{i}_{C} \xi_{R}-2 \operatorname{det}(F) \xi_{L} \cdot F^{-T} \xi_{R}\right) \\
& -\lambda \operatorname{det}(F) .
\end{aligned}
$$

\section{Existence conditions for symmetric Riemann ellipsoids}

In this section we classify symmetric relative equilibria for Dirichlet's model. We will treat the spherical case (i.e. a configuration having three equal principal axes) as a particular case of a symmetric configuration. From the singular value decomposition and the definition of the action of $G=\mathbb{Z}_{2} \ltimes(\mathrm{SO}(3) \times \mathrm{SO}(3))$ on $M=\mathrm{GL}^{+}(3)$ (or on $\mathrm{SL}^{+}(3)$ ) it follows that the stabilizer of a symmetric configuration $F$ is conjugate to the stabilizer of a diagonal configuration. That is, conjugate to $\mathbb{Z}_{2} \ltimes \mathrm{O}(2)_{\mathbf{e}}^{D}$ or $\mathbb{Z}_{2} \ltimes \mathrm{SO}(3)^{D}$ if $F$ has 2 or 1 different singular values, respectively (see [20] for a derivation of this result). Actually, if the configurations are diagonal, these are exactly their stabilizers. Here, $K^{D}$ denotes the diagonal embedding of $K \subset \mathrm{SO}(3)$ in $\mathrm{SO}(3) \times \mathrm{SO}(3)$ and $\mathrm{O}(2)_{\mathbf{e}}$ is the subgroup of $\mathrm{SO}(3)$ generated by all the rotations $R_{\theta} \in \mathrm{SO}(2)_{\mathbf{e}}$ around a given axis $\mathbf{e}$ in $\mathbb{R}^{3}$ and a rotation, $\Pi_{\mathbf{e}^{\perp}}$, by $\pi$ around an axis $\mathbf{e}^{\perp}$ perpendicular to $\mathbf{e}$. In case of the diagonal configuration $F=\operatorname{diag}(a, a, c), R_{\theta}$ is the rotation matrix by and angle $\theta$ around $(0,0,1)$ and $\Pi_{\mathbf{e}^{\perp}}$ can be chosen to be diag $(1,-1,-1)$. We introduce the following subgroups: 
- $\mathrm{SO}(2) \widetilde{\mathbf{e} \times \mathrm{SO}}(2)_{\mathbf{e}}$, generated by elements $\left(e ; R_{\theta_{1}}, R_{\theta_{2}}\right)$, with $R_{\theta_{1,2}} \in \mathrm{SO}(2)_{\mathbf{e}}$ and $\left(\sigma ; \Pi_{\mathbf{e}^{\perp}}, \Pi_{\mathbf{e}^{\perp}}\right)$,

- $\widetilde{\mathrm{O}(2)_{\mathbf{e}}}$, generated by elements $\left(e ; R_{\theta}, R_{\theta}\right)$, with $R_{\theta} \in \mathrm{SO}(2)_{\mathbf{e}}$ and $\left(\sigma ; \Pi_{\mathbf{e}^{\perp}}, \Pi_{\mathbf{e}^{\perp}}\right)$,

- $\mathbb{Z}_{2}(\mathbf{e})$, the cyclic group isomorphic to $\mathbb{Z}_{2}$ generated by the element $\left(e ; \Pi_{\mathbf{e}}, \Pi_{\mathbf{e}}\right)$.

- More generally, if $K$ is a subgroup of $\mathrm{SO}(3) \times \mathrm{SO}(3)$, we denote also by $K$ the subgroup of $\mathbb{Z}_{2} \ltimes(\mathrm{SO}(3) \times \mathrm{SO}(3))$ generated by elements $(e ; k)$, with $k \in K$.

Note that since we are going to impose the constraint $F \in \mathrm{SL}(3)$, we will consider only two kinds of symmetric configurations, especifically:

- spherical: $F=\operatorname{diag}(1,1,1)$,

- spheroidal: $F=\operatorname{diag}(a, a, c)$, with $a^{2} c=1$.

To find all the possible Riemann ellipsoids with symmetric configurations, we will have to solve (15) with $F$ of the above forms and different pairs $\left(\xi_{L}, \xi_{R}\right)$. The possible solutions are summarized in the following theorem.

Theorem 5.1. The relative equilibria for Dirichlet's model of a self-gravitating fluid mass are:

(i) The spherical equilibrium with spherical configuration $F=\operatorname{diag}(1,1,1)$, velocity-vorticity pair $(0,0)$ and Lagrange multiplier $\lambda=2 V_{1}+4 V_{2}$. Its corresponding momentum and isotropy groups are

$$
\mu=(\mathbf{j}, \mathbf{c})=(0,0), \quad G_{\mu}=\mathbb{Z}_{2} \ltimes(\mathrm{SO}(3) \times \mathrm{SO}(3)), \quad G_{F}=G_{p_{F}}=\mathbb{Z}_{2} \ltimes \mathrm{SO}(3)^{D} .
$$

(ii) The family of MacLaurin spheroids which have oblate spheroidal configurations $F=\operatorname{diag}(a, a, c)$ (with $c<a$ ) and angular velocity and vorticity parallel to the axis of symmetry $\mathbf{e}_{\mathbf{3}}$. In terms of the parameter $\Omega$ defined by $\Omega \mathbf{e}_{\mathbf{3}}=\xi_{L}-\xi_{R}$, this family is characterized by $\lambda=2\left(1-e^{2}\right)^{2 / 3} V_{1}+$ $4\left(1-e^{2}\right)^{1 / 3} V_{2}$ and the following constraint between $\Omega$ and the eccentricity $e$ :

$$
\frac{\Omega^{2}}{\pi \rho G}=2 \frac{\sqrt{1-e^{2}}}{e^{3}}\left(3-2 e^{2}\right) \arcsin e-\frac{6}{e^{2}}\left(1-e^{2}\right) .
$$

Its corresponding momentum and isotropy groups are:

$$
\begin{gathered}
\mu=(\mathbf{j}, \mathbf{c})=2\left(1-e^{2}\right)^{-1 / 3} T \Omega\left(\mathbf{e}_{\mathbf{3}},-\mathbf{e}_{\mathbf{3}}\right), \\
G_{F}=\mathbb{Z}_{2} \ltimes \mathrm{O}(2)_{\mathbf{e}_{3}}^{D}, \quad G_{\mu}=\mathrm{SO}(2)_{\mathbf{e}_{3}} \times \mathrm{SO}(2)_{\mathbf{e}_{3}}, \quad G_{p_{F}}=\widetilde{\mathrm{O}(2)_{\mathbf{e}_{3}}} .
\end{gathered}
$$


(iii) Two branches of transversal spheroids which have prolate spheroidal configurations $F=\operatorname{diag}(a, a, c)$ (with $c>a$ ). We distinguish the two branches of this family with the signs + and - . These branches are characterized by the Lagrange multiplier $\lambda=2\left(\left(1-e^{2}\right)^{1 / 3} V_{1}+\left(1-e^{2}\right)^{-1 / 3}\left(e^{2}-\right.\right.$ 2) $\left.V_{2}\right)$, the velocity-vorticity pair $\left(\xi_{L}, \xi_{R}\right)_{ \pm}=\omega_{ \pm}\left(\mathbf{n}, f_{ \pm} \mathbf{n}\right)$ with $f_{ \pm}=\frac{1 \pm e}{\sqrt{1-e^{2}}}$ (where $\mathbf{n}$ is a unit vector perpendicular to $\mathbf{e}_{\mathbf{3}}$ ) and the following constraints between $\omega_{ \pm}$and the eccentricity e:

$$
\frac{\omega_{ \pm}^{2}}{\pi \rho G}=\mp \frac{(e \mp 1)^{2}(e \pm 1)}{e^{5}}\left(3 e+\left(e^{2}-3\right) \operatorname{arctanh} e\right) .
$$

The corresponding momentum and isotropy groups are:

$$
\begin{gathered}
\mu_{ \pm}=T \omega_{ \pm}\left(-\frac{e(e \pm 2)}{\left(1-e^{2}\right)^{2 / 3}} \mathbf{n}, \pm \frac{e(e \mp 2)}{(e \mp 1)\left(1-e^{2}\right)^{1 / 6}} \mathbf{n}\right) \\
G_{F}=\mathbb{Z}_{2} \ltimes \mathrm{O}(2)_{\mathbf{e}_{3}}^{D}, \quad G_{\mu}=\operatorname{SO}(2)_{\mathbf{n}} \times \mathrm{SO}(2)_{\mathbf{n}}, \quad G_{p_{F}}=\mathbb{Z}_{2}(\mathbf{n}) .
\end{gathered}
$$

Before proving the theorem we remark that formula (32) has already been obtained by MacLaurin in 1742, as it is claimed in page 4 of Chandrasekhar's book [3].

Proof. First, using (31), (16) and (20), it is easy to see that the general conditions (15) are equivalent to

$$
\begin{aligned}
0= & 2 V_{1} F^{T}+2 V_{2}\left(I_{1} F^{T}-F^{T} F F^{T}\right)-\lambda \operatorname{det}(F) F^{-1} \\
& -T\left[\left(\left\|\xi_{L}\right\|^{2}+\left\|\xi_{R}\right\|^{2}\right) F^{T}-F^{T}\left(\xi_{L} \otimes \xi_{L}\right)-\left(\xi_{R} \otimes \xi_{R}\right) F^{T}\right. \\
& \left.+2 \operatorname{det}(F)\left(\left(F^{-1} \xi_{L} \otimes F^{-T} \xi_{R}\right)-\left(\xi_{L} \cdot F^{-T} \xi_{R}\right) F^{-1}\right)\right] \\
1= & \operatorname{det}(F)
\end{aligned}
$$

spherical case: If $F=\mathbf{I}$, then (34), (35) are simply

$$
\left(2 V_{1}+4 V_{2}-\lambda-T\left[\left\|\xi_{L}\right\|^{2}+\left\|\xi_{R}\right\|^{2}-2 \xi_{L} \cdot \xi_{R}\right]\right) \mathbf{I}+T\left[\xi_{L} \otimes \xi_{L}+\xi_{R} \otimes \xi_{R}-2 \xi_{L} \otimes \xi_{R}\right]=0
$$

Taking $\xi_{L}=\left(\xi_{L, 1}, \xi_{L, 2}, \xi_{L, 3}\right)$ and the same sorte of notation for $\xi_{R}$, the offdiagonal terms of this expression are independent of $V_{1}, V_{2}$ and $\lambda$, and equivalent to the following 6 equations:

$$
\begin{aligned}
\left(\xi_{L, 1}-\xi_{R, 1}\right)\left(\xi_{L, 2}-\xi_{R, 2}\right) & =0, & & \xi_{R, 1} \xi_{L, 2}=\xi_{R, 2} \xi_{L, 1} \\
\left(\xi_{L, 1}-\xi_{R, 1}\right)\left(\xi_{L, 3}-\xi_{R, 3}\right) & =0, & & \xi_{R, 1} \xi_{L, 3}=\xi_{R, 3} \xi_{L, 1} \\
\left(\xi_{L, 2}-\xi_{R, 2}\right)\left(\xi_{L, 3}-\xi_{R, 3}\right) & =0, & \xi_{R, 2} \xi_{L, 3} & =\xi_{R, 3} \xi_{L, 2}
\end{aligned}
$$

It follows then that $\xi_{L}=\xi_{R}$. Recall from Theorem 2.1 that the momentum of a relative equilibrium with configuration $x$ and velocity $\xi$ is given by $\mu=\mathbb{I}(x)(\xi)$. 
Then, from (25) we have $\mu=(\mathbf{j}, \mathbf{c})=(0,0)$. Therefore, $G_{F}=\mathbb{Z}_{2} \ltimes \mathrm{SO}(3)^{D}$, $G_{\mu}=G, G_{p_{F}}=G_{\mu} \cap G_{F}=G_{F}$.

Now, noting that $\left(\xi_{L}, \xi_{R}\right) \in \mathfrak{g}_{p_{F}}$ if $\xi_{L}=\xi_{R}$, and that the velocity of a relative equilibrium is defined only up to addition of elements in $\mathfrak{g}_{p_{F}}$, the relative equilibrium $\left(\mathbf{I},\left(\xi_{L}, \xi_{R}\right)\right)$ is the same as $(\mathbf{I},(0,0))$. Then the relative equilibrium conditions are satisfied with $\lambda=2 V_{1}+4 V_{2}$.

spheroidal case: We now consider $F=\operatorname{diag}(a, a, c)$ with $a^{2} c=1$. Since in the $\left\langle\mathbf{e}_{1}, \mathbf{e}_{\mathbf{2}}\right\rangle$ plane all directions are equivalent, we can assume without loss of generality that $\xi_{L, 1}=0$. Now, conditions (34), (35) are equivalent to the system

$$
\begin{aligned}
& 0=2 a V_{1}+2 a^{3} V_{2}+2 a c^{2} V_{2}-a c \lambda-a T\left(\xi_{R, 2}^{2}+\xi_{R, 3}^{2}+\xi_{L, 2}^{2}+\xi_{L, 3}^{2}\right) \\
& +2 T\left(c \xi_{R, 2} \xi_{L, 2}+a \xi_{R, 3} \xi_{L, 3}\right) \\
& 0=2 a V_{1}+2 a^{3} V_{2}+2 a c^{2} V_{2}-a c \lambda-a T\left(\xi_{R, 1}^{2}+\xi_{R, 3}^{2}+\xi_{L, 3}^{2}\right) \\
& +2 a T \xi_{R, 3} \xi_{L, 3} \\
& 0=2 c V_{1}+4 a^{2} c V_{2}-a^{2} \lambda-c T\left(\xi_{R, 1}^{2}+\xi_{R, 2}^{2}+\xi_{L, 2}^{2}\right)+2 a T \xi_{R, 2} \xi_{L, 2} \\
& 0=\xi_{R, 1} \xi_{R, 2}=\xi_{R, 1} \xi_{R, 3}=\xi_{R, 1} \xi_{L, 2}=\xi_{R, 1} \xi_{L, 3} \\
& 0=c T \xi_{R, 2} \xi_{R, 3}-2 a T \xi_{R, 3} \xi_{L, 2}+a T \xi_{L, 2} \xi_{L, 3} \\
& 0=a T \xi_{R, 2} \xi_{R, 3}-2 a T \xi_{R, 2} \xi_{L, 3}+c T \xi_{L, 2} \xi_{L, 3} \\
& 1=a^{2} c
\end{aligned}
$$

Note that these equations imply that $\xi_{R, 1}=0$. Indeed, if $\xi_{R, 1} \neq 0$ then (39) implies $\xi_{R, 2}=\xi_{R, 3}=\xi_{L, 2}=\xi_{L, 3}=0$ and so (36) and (37) imply $\xi_{R, 1}=0$ which is a contradiction. We will now proceed systematically considering four main cases: (i) $\xi_{L}=\xi_{R}=0$, (ii) $\xi_{L, 2}=0$, (iii) $\xi_{L, 3}=0$ and $\xi_{L, 2} \neq 0$, and (iv) $\xi_{L, 3} \neq 0$ and $\xi_{L, 2} \neq 0$.

(i) If $\xi_{L}=\xi_{R}=0$, then (37), (38) and (42) are the only non-trivial conditions, which are equivalent to

$$
a^{2} c=1, \quad \lambda=\frac{c}{a^{2}}\left(2 V_{1}+4 a^{2} V_{2}\right), \quad\left(a^{6}-1\right)\left(V_{1}+a^{2} V_{2}\right)=0 .
$$

As $V_{1}$ and $V_{2}$ are positive and $0<a \neq 1$ it follows from the last equation that there is no solution.

(ii) If $\xi_{L, 2}=0$, then from (36) and (37) we have $\xi_{R, 2}=0$ and so (38) and (42) give $\lambda=2 c^{2} V_{1}+4 c V_{2}$.

Let $\Omega=\xi_{L, 3}-\xi_{R, 3}$. Then the remaining non-trivial equations, (36), (37) and (42), give

$$
\Omega^{2}=\frac{2}{T} \frac{a^{2}-c^{2}}{a^{2}}\left(V_{1}+a^{2} V_{2}\right)
$$

As $V_{1}$ and $V_{2}$ are positive, then last equality implies that the spheroidal configuration $F$ is oblate, that is $a>c$. The eccentricity of the spheroid is $e^{2}=1-\frac{c^{2}}{a^{2}}$ and $a^{2}=\left(1-e^{2}\right)^{-1 / 3}$. Using the relations (16) for the partial derivatives of the self-gravitating potential, $V_{1}$ and $V_{2}$, the expression $J_{O}$ for the integrals 
$J(k, r)$ in the oblate case given by (18) and $\frac{R}{T}=2 \pi \rho G$, then the equation (43) is equivalent to

$$
\begin{aligned}
\Omega^{2} & =\frac{R}{T} e^{2}\left(J_{O}(3,2)+\left(1-e^{2}\right)^{-1 / 3} J_{O}(3,1)\right) \\
& =2 \pi \rho G e^{2}\left(J_{O}(3,2)+\left(1-e^{2}\right)^{-1 / 3} J_{O}(3,1)\right) .
\end{aligned}
$$

One can easily compute the definite integrals $J_{O}(3,2)$ and $J_{O}(3,1)$, although we avoid to display their expressions since they are quite lengthy. However the expression $J_{O}(3,2)+\left(1-e^{2}\right)^{-1 / 3} J_{O}(3,1)$ is

$$
J_{O}(3,2)+\left(1-e^{2}\right)^{-1 / 3} J_{O}(3,1)=3 \frac{e^{2}-1}{e^{4}}+\frac{3-2 e^{2}}{e^{5}} \sqrt{1-e^{2}} \arcsin e,
$$

from which (32) follows. From Corollary 4.1 it is trivial to obtain that the momentum of this relative equilibrium. Hence, using (9), the appropriate isotropy groups are also straightforward.

(iii) If $\xi_{L, 3}=0$ and $\xi_{L, 2} \neq 0$, it follows from (40) and (41) that $\xi_{R, 3}=0$. Then (37) and (42) give

$$
a^{2} c=1, \quad \lambda=2 a^{2}\left(V_{1}+\left(a^{2}+c^{2}\right) V_{2}\right) .
$$

We can set $\mathbf{n}=\mathbf{e}_{\mathbf{2}}$ and $\left(\xi_{L}, \xi_{R}\right)=\omega(\mathbf{n}, f \mathbf{n})$. So, substituting the above value of $\lambda$ into (36) and (38) these equations are

$$
\begin{aligned}
& 0=\left(a-2 c f+a f^{2}\right) T \omega^{2} \\
& 0=2\left(c^{2}-a^{2}\right) V_{1}+2 a^{2}\left(c^{2}-a^{2}\right) V_{2}-\left(c^{2} f^{2}-2 a c f+c^{2}\right) T \omega^{2}
\end{aligned}
$$

From (44) we obtain the solutions $f_{ \pm}=\frac{c \pm \sqrt{c^{2}-a^{2}}}{a}$, from which follows that the spheroids must be prolate $(c>a)$. In terms of the eccentricity $e^{2}=1-\frac{a^{2}}{c^{2}}$ we have

$$
f_{ \pm}=\frac{1 \pm e}{\sqrt{1-e^{2}}}
$$

Therefore, the equation (45) gives

$$
\left.\omega_{ \pm}^{2}=\frac{2\left(c^{2}-a^{2}\right)\left(V_{1}+a^{2} V_{2}\right)}{T\left(c^{2} f_{ \pm}^{2}-2 a c f_{ \pm}+c^{2}\right)}=\frac{1 \mp e}{T}\left(V_{1}+\left(1-e^{2}\right)^{1 / 3} V_{2}\right)\right) .
$$

Substituting in this expression $V_{1}=\frac{R}{2} J_{P}(3,2)$ and $V_{2}=\frac{R}{2} J_{P}(3,1)$, as well as $R=2 \pi \rho G T$ gives (33). As before, using the expression of the locked inertia tensor and (9) the remaining results follow.

(iv) In this case we have $\xi_{L, 2} \neq 0, \xi_{L, 3} \neq 0$ and $\xi_{L, 1}=\xi_{R, 1}=0$. Note that from (40) and (41) one should also have $\xi_{R, 2} \neq 0$ and $\xi_{R, 3} \neq 0$. So, let $\xi_{L, 2}=g \xi_{R, 2}$ and $\xi_{L, 3}=h \xi_{R, 3}$ for some reals $g, h \neq 0$. Then, using also (42), the equations (40) and (41) reduce to

$$
\begin{array}{r}
\left(1-2 a^{3} g+a^{3} g h\right) \xi_{R, 2} \xi_{R, 3}=0 \\
\left(a^{3}-2 a^{3} h+g h\right) \xi_{R, 2} \xi_{R, 3}=0 .
\end{array}
$$


These equations have solutions

$\left(h_{ \pm}, g_{ \pm}\right)=\left(\frac{5 a^{6}-1 \pm \sqrt{1-10 a^{6}+9 a^{12}}}{4 a^{6}}, \frac{1+3 a^{6} \pm \sqrt{1-10 a^{6}+9 a^{12}}}{4 a^{3}}\right)$.

Comparing (36) and (37) using $\xi_{L, 2}=g \xi_{R, 2}, \xi_{L, 3}=h \xi_{R, 3}$ and (42) we obtain $-a\left(1+g^{2}\right)+2 c g=0$ and so $g_{ \pm}$must be $\frac{1+\sqrt{1-a^{6}}}{a^{3}}$ or $\frac{1-\sqrt{1-a^{6}}}{a^{3}}$, but this is a contradiction with (46). So there is no solution for the above system.

Remark 5.1. Note that the functions $f_{+}$and $f_{-}$appearing in the characterization of transversal spheroids are inverse of each other. Therefore the two families of transversal spheroids belong to a single orbit of the symmetry group. Indeed, the $\mathbb{Z}_{2}$ symmetry interchanges the + and - families, since $\sigma \cdot\left(F ; \omega_{+} \mathbf{n}, \omega_{+} f_{+} \mathbf{n}\right)=\left(F^{T} ; \omega_{+} f_{+} \mathbf{n}, \omega_{+} \mathbf{n}\right)=\left(F ; \omega_{-} \mathbf{n}, \omega_{-} f_{-} \mathbf{n}\right)$, as it follows from their definitions that $\omega_{+}^{2} / \omega_{-}^{2}=f_{-} / f_{+}$.

\section{Remark 5.2.}

1. Theorem 5.1 is in agreement with Riemann's classification of ellipsoidal figures of equilibrium for Dirichlet's model of self-gravitating fluid masses. That is, these ellipsoidal figures of equilibrium must lie in one of the following categories: (a) the case of a uniform rotation with no internal motion (or uniform vorticity and no rotation); (b) the case when the directions of the angular velocity $\xi_{L}$ and vorticity $\xi_{R}$ are the same and coincide with a principal axis of the ellipsoid (also known as ellipsoids of type S); (c) The case when the angular velocity and vorticity are not parallel but lie in the same principal plane.

In particular, we show that for Dirichlet's model it is not possible to obtain relative equilibria with spheroidal configurations belonging to category (c).

2. The existence of transversal spheroids is referred in Chandrasekhar's book 3] (see for instance page 143), however their study is not present in the classical works of Liapunov [10] and Poincaré [18].

\section{Stability conditions for symmetric Riemann ellipsoids}

In this section we apply the singular version of the reduced energy-momentum method introduced in [21] in order to deduce the stability of the symmetric relative equilibria obtained in Theorem 5.1. In order to apply this method it is essential to compute the second derivative of the twice augmented potential $V_{\xi_{L}, \xi_{R}}^{\lambda}$. Next lemma gives that result.

Lemma 6.1. If $F$ is a critical point of the twice augmented potential

$$
V_{\left(\xi_{L}, \xi_{R}\right)}^{\lambda}=V\left(I_{1}(F), I_{2}(F)\right)-\frac{1}{2}\left[\begin{array}{ll}
\xi_{L} & \xi_{R}
\end{array}\right] \mathbb{I}(F)\left[\begin{array}{l}
\xi_{L} \\
\xi_{R}
\end{array}\right]-\lambda \operatorname{det}(F),
$$


for $\left(\xi_{L}, \xi_{R}\right) \in \mathbb{R}^{3} \times \mathbb{R}^{3}$ and $\mathbb{I}(F)$ as in Proposition 4.2 and $A, B \in T_{F} \mathrm{GL}^{+}(3)$, then

$$
\begin{aligned}
\mathbf{d}_{F}^{2} V_{\left(\xi_{L}, \xi_{R}\right)}^{\lambda}(A, B) & =\mathbf{d}_{F}^{2} V(A, B)-\frac{1}{2}\left[\begin{array}{ll}
\xi_{L} & \xi_{R}
\end{array}\right]\left(\mathbf{D}_{F}^{2} \mathbb{I}(A, B)\right)\left[\begin{array}{l}
\xi_{L} \\
\xi_{R}
\end{array}\right] \\
& -\lambda \operatorname{det}(F)\left(\operatorname{tr}\left(F^{-1} B\right) \operatorname{tr}\left(F^{-1} A\right)-\operatorname{tr}\left(F^{-1} B F^{-1} A\right)\right)
\end{aligned}
$$

where

$$
\begin{aligned}
\mathbf{d}_{F}^{2} V(A, B) & =2 \operatorname{tr}\left(B^{T} A\right)\left(V_{1}+I_{1} V_{2}\right) \\
& -2 \operatorname{tr}\left(B F^{T} F A^{T}+F B^{T} F A^{T}+F F^{T} B A^{T}\right) V_{2} \\
& +4 \operatorname{tr}\left(F^{T} A\right) \operatorname{tr}\left(F B^{T}\right)\left(V_{2}+V_{11}+2 I_{1} V_{12}+I_{1}^{2} V_{22}\right) \\
& -4 \operatorname{tr}\left(F F^{T} F B^{T}\right) \operatorname{tr}\left(F^{T} A\right)\left(V_{12}+I_{1} V_{22}\right) \\
& -4 \operatorname{tr}\left(F^{T} F F^{T} A\right) \operatorname{tr}\left(F B^{T}\right)\left(V_{12}+I_{1} V_{22}\right) \\
& +4 \operatorname{tr}\left(F^{T} F F^{T} A\right) \operatorname{tr}\left(F F^{T} F B^{T}\right) V_{22}
\end{aligned}
$$

and

$$
\left[\begin{array}{ll}
\xi_{L} & \xi_{R}
\end{array}\right] \mathbf{D}_{F}^{2} \mathbb{I}(A, B)\left[\begin{array}{l}
\xi_{L} \\
\xi_{R}
\end{array}\right]=T \operatorname{tr}\left(4 \widehat{\xi_{R}} B^{T}{\widehat{\xi_{L}}} A-2{\widehat{\xi_{L}}}^{2} A B^{T}-2{\widehat{\xi_{R}}}^{2} B^{T} A\right)
$$

Proof. We will just sketch the computation of $\mathbf{d}_{F}^{2} V(A, B)$.

Recall from the proof of Theorem [5.1, that

$$
\mathbf{d} V(F) \cdot A=2\left(V_{1}+I_{1} V_{2}\right) \operatorname{tr}\left(F^{T} A\right)-2 V_{2} \operatorname{tr}\left(F^{T} F F^{T} A\right) .
$$

Differentiating again using the expressions for $\mathbf{d} I_{1}(F) \cdot A$ and $\mathbf{d} I_{2}(F) \cdot A$ given in (21) and (22) and the chain rule the result follows.

For the expression $\mathbf{D}_{F}^{2} \mathbb{I}(A, B)$ we differentiate the expression (24), which in this case takes the form

$$
\left\langle\left(\widehat{\xi_{L}}, \widehat{\xi_{R}}\right), \mathbb{I}(F)\left(\widehat{\xi_{L}}, \widehat{\xi_{R}}\right)\right\rangle=T \operatorname{tr}\left[2{\widehat{\xi_{R}}}^{T}{\widehat{\xi_{L}}} F-F^{T} \widehat{F}^{2}-F F^{T}{\widehat{\xi_{L}}}^{2}\right]
$$

Then, applying standard properties of the trace, we get

$$
\left\langle\left(\widehat{\xi_{L}}, \widehat{\xi_{R}}\right),(\mathbf{D I}(F) \cdot A)\left(\widehat{\xi_{L}}, \widehat{\xi_{R}}\right)\right\rangle=T \operatorname{tr}\left[4 \widehat{\xi_{R}} A^{T} \widehat{\xi_{L}} F-2 A^{T} \widehat{F}^{2}-2 A F^{T}{\widehat{\xi_{L}}}^{2}\right] \text {. }
$$

The expression for $\mathbf{D}_{F}^{2} \mathbb{I}(A, B)$ stated follows now easily. Finally, using (23) to differenciate the expression

$$
\mathbf{d} \operatorname{det}(F) \cdot A=\operatorname{det}(F) \operatorname{tr}\left(F^{-1} A\right)
$$

we get

$$
\mathbf{d}_{F}^{2}(\operatorname{det}(F))(A, B)=\operatorname{det}(F)\left[\operatorname{tr}\left(F^{-1} B\right) \operatorname{tr}\left(F^{-1} A\right)-\operatorname{tr}\left(F^{-1} B F^{-1} A\right)\right] .
$$




\subsection{Spherical equilibrium}

We now study the stability of the spherical equilibrium. Notice from the outline of the method in Section 3 that for this equilibrium whe have that $\mathfrak{q}^{\mu}$, the correction term and the Arnold form are all trivial, as well as the velocity-vorticity pair $\left(\xi_{L}, \xi_{R}\right)$. As a consequence, $\Sigma_{\text {int }}^{\mathrm{SL}(3)}=\mathbf{S}^{\mathrm{SL}(3)}$, the orthogonal complement to the $G$-orbit at the identity in $\mathrm{SL}(3)$. Hence, to conclude stability of the spherical equilibrium we need to study the definiteness of

$$
\left.\mathbf{d}_{\mathbf{I}}^{2} V_{(0,0)}^{\lambda}\right|_{\mathbf{S}^{\mathrm{SL}(3)}} .
$$

Theorem 6.1. For Dirichlet's model, the spherical equilibrium is nonlinearly G-stable.

Proof. Recall that $T_{\mathbf{I}} \mathrm{SL}(3)$ is the space of traceless matrices. Also, the infinitesimal action of $\mathfrak{g}$ on $\mathrm{GL}^{+}(3)$ at $\mathbf{I}$ is $\left(\xi_{L}, \xi_{R}\right)_{\mathrm{GL}^{+}(3)}(\mathbf{I})=\widehat{\xi_{L}}-\widehat{\xi_{R}}$. Then

$$
\mathbf{S}^{\mathrm{SL}(3)}=\left\{A \in T_{\mathbf{I}} \mathrm{SL}(3): \operatorname{tr}\left(A \widehat{\xi_{L}}-A \widehat{\xi_{R}}\right)=0 \quad \forall \xi_{L}, \xi_{R} \in \mathbb{R}^{3}\right\} .
$$

Therefore $\mathbf{S}^{\mathrm{SL}(3)}$ is the space of traceless symmetric matrices. That is, matrices of the form

$$
A=\left[\begin{array}{ccc}
a_{11} & a_{12} & a_{13} \\
a_{12} & a_{22} & a_{23} \\
a_{13} & a_{23} & -\left(a_{11}+a_{22}\right)
\end{array}\right] .
$$

We fix a basis for $\mathbf{S}^{\mathrm{SL}(3)}$ with respect to which the components of $A$ are $\left(a_{11}, a_{22}, a_{12}, a_{13}, a_{23}\right)$.

By Lemma 6.1, the expression of $\left.\mathbf{d}_{\mathbf{I}}^{2} V_{(0,0)}^{\lambda}\right|_{\mathbf{S}^{\mathrm{SL}(3)}}$, with $\lambda=2 V_{1}+4 V_{2}$ as given in Theorem 5.1, reduces to:

$$
\left.\mathbf{d}_{\mathbf{I}}^{2} V_{(0,0)}^{\lambda}\right|_{\mathbf{S}^{\mathrm{SL}(3)}}(A, B)=4\left(V_{1}+V_{2}\right) \operatorname{tr}(B A),
$$

where we have applied the fact that $A$ and $B$ are traceless symmetric matrices. Therefore in this basis we have

$$
\left.\mathbf{d}_{\mathbf{I}}^{2} V_{(0,0)}^{\lambda}\right|_{\mathbf{S}^{\mathrm{SL}(3)}}=4\left(V_{1}+V_{2}\right)\left[\begin{array}{ccccc}
2 & 1 & 0 & 0 & 0 \\
1 & 2 & 0 & 0 & 0 \\
0 & 0 & 2 & 0 & 0 \\
0 & 0 & 0 & 2 & 0 \\
0 & 0 & 0 & 0 & 2
\end{array}\right]
$$

As $V_{1}+V_{2}$ is positive, the eigenvalues of this matrix are $12\left(V_{1}+V_{2}\right), 4\left(V_{1}+V_{2}\right)$ and $8\left(V_{1}+V_{2}\right)$ with multiplicities 1,1 and 3 respectively. These are all positive, therefore the spherical equilibrium is $G$-stable.

\subsection{MacLaurin spheroids}

We now study the nonlinear stability of MacLaurin spheroids in the setup of previous sections (Theorem 5.1). As it has been stated, a MacLaurin spheroid 
has an oblate configuration which, with no loss of generality, we suppose diagonal. This configuration is uniquely characterized by the eccentricity $e \in(0,1)$. In order to apply Theorem 3.1 one needs first to split $\mathfrak{g}=\mathbb{R}^{3} \oplus \mathbb{R}^{3}$ according to (10), that is as

$$
\mathfrak{g}_{\mu}=\mathfrak{g}_{p_{F}} \oplus \mathfrak{p} \quad \text { and } \quad \mathfrak{g}=\mathfrak{g}_{F} \oplus \mathfrak{p} \oplus \mathfrak{t} .
$$

Recall that for the MacLaurin spheroid one has $G_{F}=\mathbb{Z}_{2} \ltimes \mathrm{O}(2)^{D}$ and $G_{\mu}=$ $\mathrm{SO}(2)_{\mathbf{e}_{3} \times \mathrm{SO}}(2)_{\mathbf{e}_{3}}$. One can then choose the following ordered orthonormal bases (with respect to the Euclidean product in $\mathbb{R}^{3} \oplus \mathbb{R}^{3}$ ) for each of the spaces of the above splitting: If we define $h=\frac{1}{\sqrt{2}}\left(\mathbf{e}_{3}, \mathbf{e}_{3}\right), p=\frac{1}{\sqrt{2}}\left(\mathbf{e}_{\mathbf{3}},-\mathbf{e}_{\mathbf{3}}\right), t_{1}=$ $\left(\mathbf{e}_{1}, 0\right), t_{2}=\left(0, \mathbf{e}_{1}\right), t_{3}=\left(\mathbf{e}_{2}, 0\right), t_{4}=\left(0, \mathbf{e}_{2}\right)$, then

$$
\begin{aligned}
\mathfrak{g}_{F} & =\operatorname{span}\{h\} \\
\mathfrak{p} & =\operatorname{span}\{p\} \\
\mathfrak{t} & =\operatorname{span}\left\{t_{1}, t_{2}, t_{3}, t_{4}\right\} .
\end{aligned}
$$

It is straightforward to check that these subspaces are invariant for $G_{P_{F}}=$ $\widetilde{\mathrm{O}(2)_{\mathbf{e}_{3}}}$. The orthogonal velocity $\xi^{\perp}$ for the MacLaurin relative equilibrium is the orthogonal projection of the velocity $\xi$ onto $\mathfrak{p}$. Then,

$$
\begin{aligned}
\left(\xi_{L}, \xi_{R}\right)^{\perp} & =\frac{1}{2}\left(\xi_{L, 3} \mathbf{e}_{\mathbf{3}}, \xi_{R, 3} \mathbf{e}_{\mathbf{3}}\right) \cdot\left(\mathbf{e}_{\mathbf{3}},-\mathbf{e}_{\mathbf{3}}\right)\left(\mathbf{e}_{\mathbf{3}},-\mathbf{e}_{\mathbf{3}}\right) \\
& =\frac{1}{2}\left(\xi_{L, 3}-\xi_{R, 3}\right)\left(\mathbf{e}_{\mathbf{3}},-\mathbf{e}_{\mathbf{3}}\right)=\frac{\Omega}{2}\left(\mathbf{e}_{\mathbf{3}},-\mathbf{e}_{\mathbf{3}}\right)=\frac{\Omega}{\sqrt{2}} p
\end{aligned}
$$

where $\Omega$ must satisfy (32). As already defined, $\widehat{\mathbb{I}}_{0}$ is the restriction of $\mathbb{I}$ to $\mathfrak{p} \oplus \mathfrak{t}$. The locked inertia matrix for the configuration $F=\operatorname{diag}(a, a, c)$ is, according to 25 ,

$$
\mathbb{I}(F)=T\left[\begin{array}{ll}
D_{1} & D_{2} \\
D_{2} & D_{1}
\end{array}\right]
$$

where $D_{1}=\operatorname{diag}\left(a^{2}+c^{2}, a^{2}+c^{2}, 2 a^{2}\right)$ and $D_{2}=-\operatorname{diag}\left(2 a c, 2 a c, 2 a^{2}\right)$. It is now straighforward to obtain the $\widehat{\mathbb{I}}_{0}$ matrix with respect to the basis $\left(p, t_{1}, t_{2}, t_{3}, t_{4}\right)$. That is

$$
\widehat{\mathbb{I}}_{0}=T\left[\begin{array}{ccccc}
4 a^{2} & 0 & 0 & 0 & 0 \\
0 & a^{2}+c^{2} & -2 a c & 0 & 0 \\
0 & -2 a c & a^{2}+c^{2} & 0 & 0 \\
0 & 0 & 0 & a^{2}+c^{2} & -2 a c \\
0 & 0 & 0 & -2 a c & a^{2}+c^{2}
\end{array}\right]
$$

Or, in terms of the eccentricity

$$
\widehat{\mathbb{I}}_{0}=T\left[\begin{array}{ccccc}
\frac{4}{\sqrt[3]{1-e^{2}}} & 0 & 0 & 0 & 0 \\
0 & \frac{2-e^{2}}{\sqrt[3]{1-e^{2}}} & -2 \sqrt[6]{1-e^{2}} & 0 & 0 \\
0 & -2 \sqrt[6]{1-e^{2}} & \frac{2-e^{2}}{\sqrt[3]{1-e^{2}}} & 0 & 0 \\
0 & 0 & 0 & \frac{2-e^{2}}{\sqrt[3]{1-e^{2}}} & -2 \sqrt[6]{1-e^{2}} \\
0 & 0 & 0 & -2 \sqrt[6]{1-e^{2}} & \frac{2-e^{2}}{\sqrt[3]{1-e^{2}}}
\end{array}\right]
$$


We can use $\widehat{\mathbb{I}}_{0}$ and $\left(\xi_{L}, \xi_{R}\right)^{\perp}$ to compute the momentum of a MacLaurin spheroid. Indeed

$$
\mu=\widehat{\mathbb{I}}_{0}\left(\xi_{L}, \xi_{R}\right)^{\perp}=\frac{\Omega}{\sqrt{2}} \widehat{\mathbb{I}}_{0}(p)=\frac{2 \sqrt{2} T \Omega}{\left(1-e^{2}\right)^{1 / 3}} p
$$

which is of course the same as the value obtained in Theorem 5.1 under the identification $\mathfrak{g} \simeq \mathfrak{g}^{*}$ induced by the Euclidean product in $\mathbb{R}^{3} \oplus \mathbb{R}^{3}$.

In order to apply Theorem 3.1 we need to verify that the singular Arnold form is nondegenerate.

Proposition 6.1. For a MacLaurin spheroid $\mathfrak{q}^{\mu}=\mathfrak{t}$ and the Arnold form, defined in (14), is positive definite for all eccentricities.

Proof. Recall that the Arnold form Ar: $\mathfrak{q}^{\mu} \times \mathfrak{q}^{\mu} \rightarrow \mathbb{R}$ is defined by:

$$
\operatorname{Ar}\left(\gamma_{1}, \gamma_{2}\right)=\left\langle\operatorname{ad}_{\gamma_{1}}^{*} \mu, \Lambda(F, \mu)\left(\gamma_{2}\right)\right\rangle,
$$

where

$$
\Lambda(F, \mu)(\gamma)=\widehat{\mathbb{I}}_{0}^{-1}\left(\operatorname{ad}_{\gamma}^{*} \mu\right)+\mathbb{P}_{\mathfrak{p}^{*} \oplus \mathfrak{t}^{*}}\left[\operatorname{ad}_{\gamma}\left(\widehat{\mathbb{I}}_{0}^{-1} \mu\right)\right]
$$

First, we compute the space $\mathfrak{q}^{\mu}$. Notice the following relations for the adjoint representation of $G$ :

$$
\operatorname{ad}_{t_{1}} p=\frac{-1}{\sqrt{2}} t_{3}, \quad \operatorname{ad}_{t_{2}} p=\frac{1}{\sqrt{2}} t_{4}, \quad \operatorname{ad}_{t_{3}} p=\frac{1}{\sqrt{2}} t_{1}, \quad \operatorname{ad}_{t_{4}} p=\frac{-1}{\sqrt{2}} t_{2},
$$

and

$$
\begin{aligned}
\operatorname{ad}_{t_{1}} t_{2} & =\operatorname{ad}_{t_{1}} t_{4}=\operatorname{ad}_{t_{2}} t_{3}=\operatorname{ad}_{t_{3}} t_{4}=0, \\
\operatorname{ad}_{t_{1}} t_{3} & =\frac{1}{\sqrt{2}}(h+p), \\
\operatorname{ad}_{t_{2}} t_{4} & =\frac{1}{\sqrt{2}}(h-p) .
\end{aligned}
$$

Also, recall that under our identification $\mathfrak{g} \simeq \mathfrak{g}^{*}$ we have $\operatorname{ad}_{\gamma}^{*} \rho=-\operatorname{ad}_{\gamma} \rho$, for $\gamma, \in \mathfrak{g}, \rho \in \mathfrak{g}^{*}$, and where $\rho$ in the right hand side is identified with an element of $\mathfrak{g}$. Therefore $\mathbb{P}_{\mathfrak{g}_{F}}\left(\operatorname{ad}_{t_{i}}^{*} \mu\right)=0$ for $i=1,2,3,4$, hence $\mathfrak{q}^{\mu}=\mathfrak{t}$. As $\frac{\Omega}{\sqrt{2}} \widehat{\mathbb{I}}_{0}(p)=\mu$ then $\widehat{\mathbb{I}}_{0}^{-1}(\mu)=\frac{\Omega}{\sqrt{2}} p$. Then, from (48) we obtain

$$
\begin{aligned}
\operatorname{ad}_{t_{1}}\left(\widehat{\mathbb{I}}_{0}^{-1} \mu\right) & =-\frac{\Omega}{2} t_{3} & \operatorname{ad}_{t_{2}}\left(\widehat{\mathbb{I}}_{0}^{-1} \mu\right) & =\frac{\Omega}{2} t_{4} \\
\operatorname{ad}_{t_{3}}\left(\widehat{\mathbb{I}}_{0}^{-1} \mu\right) & =\frac{\Omega}{2} t_{1} & \operatorname{ad}_{t_{4}}\left(\widehat{\mathbb{I}}_{0}^{-1} \mu\right) & =-\frac{\Omega}{2} t_{2}
\end{aligned}
$$

The inverse of the matrix (47) is not difficult to compute. Here we just state 
the values of $\widehat{\mathbb{I}}_{0}^{-1}\left(\operatorname{ad}_{w}^{*} \mu\right)=-\widehat{\mathbb{I}}_{0}^{-1}\left(\operatorname{ad}_{w} \mu\right)$ on vectors $w$ of the fixed basis:

$$
\begin{aligned}
& \widehat{\mathbb{I}}_{0}^{-1}\left(\operatorname{ad}_{t_{1}}^{*} \mu\right)=\frac{2 T \Omega}{\left(1-e^{2}\right)^{1 / 3}} \widehat{\mathbb{I}}_{0}^{-1}\left(t_{3}\right)=\frac{-2\left(e^{2}-2\right) \Omega}{e^{4}} t_{3}+\frac{4 \sqrt{1-e^{2}} \Omega}{e^{4}} t_{4} \\
& \widehat{\mathbb{I}}_{0}^{-1}\left(\operatorname{ad}_{t_{2}}^{*} \mu\right)=-\frac{2 T \Omega}{\left(1-e^{2}\right)^{1 / 3}} \widehat{\mathbb{I}}_{0}^{-1}\left(t_{4}\right)=\frac{-4 \sqrt{1-e^{2}} \Omega}{e^{4}} t_{3}+\frac{2\left(e^{2}-2\right) \Omega}{e^{4}} t_{4} \\
& \widehat{\mathbb{I}}_{0}^{-1}\left(\operatorname{ad}_{t_{3}}^{*} \mu\right)=-\frac{2 T \Omega}{\left(1-e^{2}\right)^{1 / 3}} \widehat{\mathbb{I}}_{0}^{-1}\left(t_{1}\right)=\frac{2\left(e^{2}-2\right) \Omega}{e^{4}} t_{1}-\frac{4 \sqrt{1-e^{2}} \Omega}{e^{4}} t_{2} \\
& \widehat{\mathbb{I}}_{0}^{-1}\left(\operatorname{ad}_{t_{4}}^{*} \mu\right)=\frac{2 T \Omega}{\left(1-e^{2}\right)^{1 / 3}} \widehat{\mathbb{I}}_{0}^{-1}\left(t_{2}\right)=\frac{4 \sqrt{1-e^{2}} \Omega}{e^{4}} t_{1}-\frac{2\left(e^{2}-2\right) \Omega}{e^{4}} t_{2}
\end{aligned}
$$

From these expressions it follows easily that

$$
\begin{aligned}
& \Lambda(F, \mu)\left(t_{1}\right)=-\frac{e^{4}+4 e^{2}-8}{2 e^{4}} \Omega t_{3}+\frac{4 \sqrt{1-e^{2}}}{e^{4}} \Omega t_{4} \\
& \Lambda(F, \mu)\left(t_{2}\right)=\frac{-4 \sqrt{1-e^{2}}}{e^{4}} \Omega t_{3}+\frac{e^{4}+4 e^{2}-8}{2 e^{4}} \Omega t_{4} \\
& \Lambda(F, \mu)\left(t_{3}\right)=\frac{e^{4}+4 e^{2}-8}{2 e^{4}} \Omega t_{1}-\frac{4 \sqrt{1-e^{2}}}{e^{4}} \Omega t_{2} \\
& \Lambda(F, \mu)\left(t_{4}\right)=\frac{4 \sqrt{1-e^{2}}}{e^{4}} \Omega t_{1}-\frac{e^{4}+4 e^{2}-8}{2 e^{4}} \Omega t_{2}
\end{aligned}
$$

Finally, the entries of the Arnold matrix are given by

$$
\operatorname{Ar}\left(t_{i}, t_{j}\right)=\left\langle\mu, \operatorname{ad}_{t_{i}} \Lambda(F, \mu)\left(t_{j}\right)\right\rangle=\frac{2 \sqrt{2} T \Omega}{\left(1-e^{2}\right)^{1 / 3}}\left\langle p, \operatorname{ad}_{t_{i}} \Lambda(F, \mu)\left(t_{j}\right)\right\rangle, \quad i=1,2,3,4 .
$$

Using (49) the Arnold matrix is then given by

$$
\operatorname{Ar}=\left[\begin{array}{cccc}
A_{1} & -A_{2} & 0 & 0 \\
-A_{2} & A_{1} & 0 & 0 \\
0 & 0 & A_{1} & -A_{2} \\
0 & 0 & -A_{2} & A_{1}
\end{array}\right] \quad \text { with } \quad\left\{\begin{array}{l}
A_{1}=\frac{\left(8-e^{4}-4 e^{2}\right) T \Omega^{2}}{e^{4}\left(1-e^{2}\right)^{\frac{1}{3}}} \\
A_{2}=\frac{8\left(1-e^{2}\right)^{\frac{1}{6}} T \Omega^{2}}{e^{4}}
\end{array}\right.
$$

The trace and the determinant of each block of Ar are positive and so Ar is positive definite.

Next theorem gives the stability of the MacLaurin spheroids.

Theorem 6.2. A MacLaurin spheroid with eccentricity e and momentum $\mu$ is:

(i) nonlinearly $G_{\mu}$-stable if $e<e_{0}$ with $e_{0} \simeq 0.952887$.

(ii) unstable if $e>e_{0}$. 
Proof. For (i): As the Arnold form is non-degenerate, $G_{\mu}$-stability will follow whenever $\left.\left(\mathbf{d}_{F}^{2} V_{\xi^{\perp}}+\operatorname{corr}_{\xi^{\perp}}(F)\right)\right|_{\Sigma_{\text {int }}}$ is positive definite. For, recall from (12) that

$\Sigma_{\mathrm{int}}=\left\{\gamma_{\mathrm{SL}(3)}(F)+A: \gamma \in \mathfrak{q}^{\mu}, A \in \mathbf{S}^{\mathrm{SL}(3)}\right.$, and $\left.\left(\mathbf{D I}(F) \cdot\left(\gamma_{\mathrm{SL}(3)}(F)+A\right)\right)\left(\xi^{\perp}\right) \in \mathfrak{p}^{*}\right\}$,

where $\mathbf{S}^{\mathrm{SL}(3)}$ is the linear slice for the $G$-action on $\mathrm{SL}(3)$ at the oblate configuration, $F=\operatorname{diag}(a, a, c)$. Matrices $A \in \mathbf{S}^{\mathrm{SL}(3)}$ must verify

$$
\begin{aligned}
& 0=\operatorname{tr}\left[A^{T}(\widehat{\xi} F-F \widehat{\eta})\right], \quad \forall \xi, \eta \in \mathbb{R}^{3}, \quad \text { and } \\
& 0=\operatorname{tr}\left(F^{-1} A\right)
\end{aligned}
$$

because $A$ must belong, respectively to the orthogonal complement to the tangent space to the group orbit through $F$ and $A \in T_{F} \mathrm{SL}(3)$. These two conditions give that $A$ must be of the form

$$
A=\left[\begin{array}{ccc}
a_{11} & a_{12} & 0 \\
a_{12} & a_{22} & 0 \\
0 & 0 & a_{33}
\end{array}\right] \quad \text { with } \quad \frac{1}{a}\left(a_{11}+a_{22}\right)+\frac{1}{c} a_{33}=0 .
$$

Therefore we can describe $\mathbf{S}^{\mathrm{SL}(3)}$ as the set of matrices of the form

$$
A=\left[\begin{array}{ccc}
a_{1}+a_{2} & a_{3} & 0 \\
a_{3} & a_{1}-a_{2} & 0 \\
0 & 0 & -2 \frac{c}{a} a_{1}
\end{array}\right]
$$

with $a_{1}, a_{2}, a_{3} \in \mathbb{R}$. Let the vector $\gamma \in \mathfrak{q}^{\mu}=\mathfrak{t}$ be $\gamma=\left(\gamma_{1}, \gamma_{2}, \gamma_{3}, \gamma_{4}\right)$ with respect to the basis $\left(t_{1}, t_{2}, t_{3}, t_{4}\right)$. Therefore, using (8), we have

$$
\gamma_{\mathrm{SL}(3)}(F)+A=\left[\begin{array}{ccc}
a_{1}+a_{2} & a_{3} & c \gamma_{3}-a \gamma_{4} \\
a_{3} & a_{1}-a_{2} & -c \gamma_{1}+a \gamma_{2} \\
-a \gamma_{3}+c \gamma_{4} & a \gamma_{1}-c \gamma_{2} & -2 \frac{c}{a} a_{1}
\end{array}\right] .
$$

The set $\Sigma_{\text {int }}$ is precisely the set of matrices $\lambda_{\mathrm{SL}(3)}(F)+A$ for which

$$
\left(\mathbf{D I}(F) \cdot\left(\gamma_{\mathrm{SL}(3)}(F)+A\right)\right)\left(\xi_{L}, \xi_{R}\right)^{\perp} \in \mathfrak{p}^{*}=\left(\mathfrak{g}_{F}+\mathfrak{t}\right)^{\circ},
$$

where $(\mathfrak{h}+\mathfrak{t})^{\circ}$ denotes the annihilator of $\mathfrak{h}+\mathfrak{t}$. Using $\left(\xi_{L}, \xi_{R}\right)^{\perp}=\frac{\Omega}{\sqrt{2}} p$ and $w \in\left\{h, t_{1}, t_{2}, t_{3}, t_{4}\right\}$ and differentiating (24), the computation of

$$
\left\langle w,\left(\mathbf{D} \mathbb{I}(F) \cdot\left(\lambda_{\mathrm{SL}(3)}(F)+A\right)\right)\left(\xi_{L}, \xi_{+} R\right)^{\perp}\right\rangle
$$


gives, in terms of the eccentricity $e$ :

$$
\begin{aligned}
\left\langle h,\left(\mathbf{D} \mathbb{I}(F) \cdot\left(\gamma_{\mathrm{SL}(3)}(F)+A\right)\right)\left(\xi_{L}, \xi_{R}\right)^{\perp}\right\rangle & =0 \\
\left\langle t_{1},\left(\mathbf{D} \mathbb{I}(F) \cdot\left(\gamma_{\mathrm{SL}(3)}(F)+A\right)\right)\left(\xi_{L}, \xi_{R}\right)^{\perp}\right\rangle & =\frac{T \Omega}{2\left(1-e^{2}\right)^{1 / 3}}\left[\left(2+e^{2}\right) \gamma_{3}-2 \sqrt{1-e^{2}} \gamma_{4}\right] \\
\left\langle t_{2},\left(\mathbf{D} \mathbb{I}(F) \cdot\left(\gamma_{\mathrm{SL}(3)}(F)+A\right)\right)\left(\xi_{L}, \xi_{R}\right)^{\perp}\right\rangle & =\frac{T \Omega}{2\left(1-e^{2}\right)^{1 / 3}}\left[2 \sqrt{1-e^{2}} \gamma_{3}-\left(2+e^{2}\right) \gamma_{4}\right] \\
\left\langle t_{3},\left(\mathbf{D} \mathbb{I}(F) \cdot\left(\gamma_{\mathrm{SL}(3)}(F)+A\right)\right)\left(\xi_{L}, \xi_{R}\right)^{\perp}\right\rangle & =\frac{T \Omega}{2\left(1-e^{2}\right)^{1 / 3}}\left[-\left(2+e^{2}\right) \gamma_{1}+2 \sqrt{1-e^{2}} \gamma_{2}\right] \\
\left\langle t_{4},\left(\mathbf{D} \mathbb{I}(F) \cdot\left(\gamma_{\mathrm{SL}(3)}(F)+A\right)\right)\left(\xi_{L}, \xi_{R}\right)^{\perp}\right\rangle & =\frac{T \Omega}{2\left(1-e^{2}\right)^{1 / 3}}\left[-2 \sqrt{1-e^{2}} \gamma_{1}+\left(2+e^{2}\right) \gamma_{2}\right]
\end{aligned}
$$

It follows from the above expressions that

$$
\left(\mathbf{D} \mathbb{I}(F) \cdot\left(\lambda_{\mathrm{SL}(3)}(F)+A\right)\right)\left(\xi_{L}, \xi_{R}\right)^{\perp} \in\left(\mathfrak{g}_{F}+\mathfrak{t}\right)^{\circ}
$$

if and only if $\gamma=0$. This is equivalent to $\Sigma_{\mathrm{int}}=\mathbf{S}^{\mathrm{SL}(3)}$. Let us now compute the correction term restricted to $\Sigma_{\mathrm{int}}=\mathbf{S}^{\mathrm{SL}(3)}$. For any $A \in \mathbf{S}^{\mathrm{SL}(3)}$ one has $(\mathbf{D I}(F) \cdot A)\left(\xi_{L}, \xi_{R}\right)^{\perp}=\frac{4 T \Omega \sqrt{2}}{\left(1-e^{2}\right)^{1 / 6}} a_{1} p$ and so

$$
\mathbb{P}_{\mathbf{t}^{*} \oplus \mathfrak{p}^{*}}\left[(\mathbf{D} \mathbb{I}(F) \cdot A)\left(\xi_{L}, \xi_{R}\right)^{\perp}\right]=\left(\frac{4 T \Omega \sqrt{2}}{\left(1-e^{2}\right)^{1 / 6}} a_{1}, 0,0,0,0\right) .
$$

From the expression of $\widehat{\mathbb{I}}_{0}$ it is straightforward to obtain

$$
\widehat{\mathbb{I}}_{0}^{-1}\left(\mathbb{P}_{\mathbf{t}^{*} \oplus \mathfrak{p}^{*}}\left[(\mathbf{D} \mathbb{I}(F) \cdot B)\left(\xi_{L}, \xi_{R}\right)^{\perp}\right]\right)=\sqrt{2} \Omega\left(1-e^{2}\right)^{1 / 6} b_{1} p,
$$

where $b_{1}$ is the entry of $B \in \mathbf{S}^{\mathrm{SL}(3)}$ playing the same role of $a_{1}$ in $A$. Then, from (13)

$$
\operatorname{corr}_{\left(\xi_{L}, \xi_{R}\right)^{\perp}}(F)(A, B)=\left\langle\frac{4 T \Omega \sqrt{2}}{\left(1-e^{2}\right)^{1 / 6}} a_{1} p, \sqrt{2} \Omega\left(1-e^{2}\right)^{1 / 6} b_{1} p\right\rangle=8 T \Omega^{2} a_{1} b_{1} .
$$

The computation of $\mathbf{d}_{F}^{2} V_{\left(\xi_{L}, \xi_{R}\right)^{\perp}}^{\lambda}(A, B)$ is lengthy but with no difficulties. Using Lemma 6.1 we obtain

$$
\begin{aligned}
\left\langle\left(\xi_{L}, \xi_{R}\right)^{\perp},\left(\mathbf{D}_{F}^{2} \mathbb{I}(A, B)\right)\left(\xi_{L}, \xi_{R}\right)^{\perp}\right\rangle & =4 a_{1} b_{1} T \Omega^{2} \\
\mathbf{d}_{F}^{2} \operatorname{det}(A, B) & =-2 c\left(3 a_{1} b_{1}+a_{2} b_{2}+a_{3} b_{3}\right)
\end{aligned}
$$

We fix a basis for the slice $\mathrm{S}^{\mathrm{SL}(3)}$ in which the coordinates of $A$ in (50) are $A=\left(a_{1}, a_{2}, a_{3}\right)$. With respect to this basis the matrix for $\left.\mathbf{d}_{F}^{2} V\right|_{\Sigma_{\text {int }}}$ is given by $\left.\mathbf{d}_{F}^{2} V\right|_{\Sigma_{\text {int }}}=\operatorname{diag}\left(D_{1}, D_{2}, D_{2}\right)$ with

$$
\begin{aligned}
\frac{a^{10}}{4} D_{1}= & a^{4}\left(a^{6}+2\right) V_{1}+3 a^{6}\left(a^{6}-1\right) V_{2}+4\left(a^{6}-1\right)^{2} V_{11}+8 a^{2}\left(a^{6}-1\right)^{2} V_{12} \\
& +4 a^{4}\left(a^{6}-1\right)^{2} V_{22}, \\
\frac{a^{10}}{4} D_{2}= & a^{10} V_{1}+a^{6}\left(1-a^{6}\right) V_{2} .
\end{aligned}
$$


Therefore, with respect to this basis we have

$\left.\left(\mathbf{d}_{F}^{2} V_{\xi^{\perp}}^{\lambda}+\operatorname{corr}_{\xi^{\perp}}(F)\right)\right|_{\Sigma_{\text {int }}}=\operatorname{diag}\left(D_{1}+6 c \lambda+6 T \Omega^{2}, D_{2}+2 c \lambda, D_{2}+2 c \lambda\right)=\operatorname{diag}\left(S_{1}, S_{2}, S_{2}\right)$.

For the MacLaurin spheroid we have, from Theorem 5.1, $\lambda=2\left(c^{2} V_{1}+2 c V_{2}\right)$ and $\Omega^{2}=\frac{2}{T} e^{2}\left(V_{1}+a^{2} V_{2}\right)$. Then, in terms of the eccentricity, we have

$$
\begin{aligned}
& S_{1}=\frac{8}{1-e^{2}}\left(\left(3-4 e^{2}+e^{4}\right) V_{1}+3\left(1-e^{2}\right)^{2 / 3} V_{2}+2 e^{4}\left(1-e^{2}\right)^{2 / 3} V_{11}\right. \\
& \left.\quad+4 e^{4}\left(1-e^{2}\right)^{1 / 3} V_{12}+2 e^{4} V_{22}\right) \\
& S_{2}=\frac{4}{1-e^{2}}\left(\left(2-3 e^{2}+e^{4}\right) V_{1}+\left(2-3 e^{2}\right)\left(1-e^{2}\right)^{2 / 3} V_{2}\right)
\end{aligned}
$$

Expressing the partial derivatives of $V$ in terms of the integrals $J_{O}(k, r)$ we obtain

$$
\begin{aligned}
& S_{1}=\frac{2 R}{e^{5}}\left(9 e\left(3-5 e^{2}+2 e^{4}\right)-\sqrt{1-e^{2}}\left(27-36 e^{2}+8 e^{4}\right) \arcsin e\right) \\
& S_{2}=\frac{R}{e^{5}}\left(\left(1-e^{2}\right) e\left(3+4 e^{2}\right)-\sqrt{1-e^{2}}\left(3+2 e^{2}-4 e^{4}\right) \arcsin e\right)
\end{aligned}
$$

The plots of $S_{1}$ and $S_{2}$ are shown in Figure 1. They show that $S_{1}$ is always positive in $(0,1)$ while $S_{2}$ has a root $e_{0} \in(0,1)$ being positive for $e<e_{0}$ and negative for $e>e_{0}$. We used the Mathematica programing system for the numerical computation of $e_{0}$ to obtain $e_{0} \simeq 0.952887$. Therefore MacLaurin spheroids are $G_{\mu}$-stable for $e<e_{0}$.
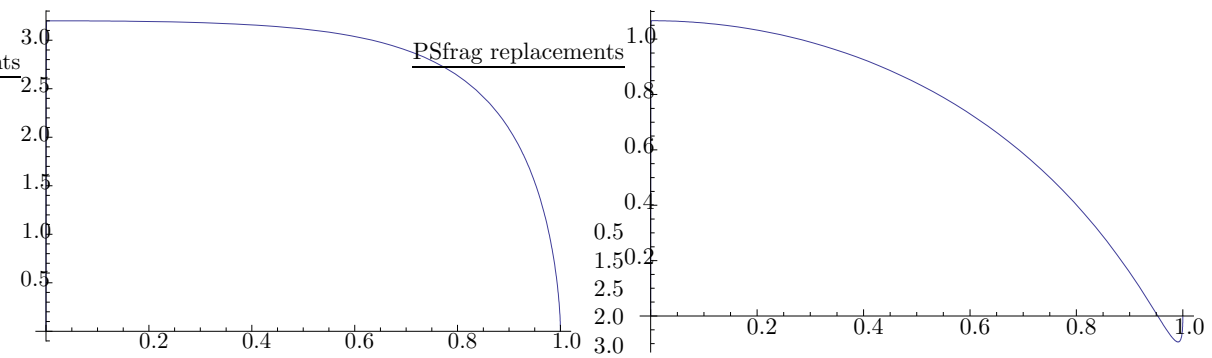

Figure 1: The plots of $S_{1}$ (left) and $S_{2}$ (right) in $R$ units.

For (ii): When $e \in\left[e_{0}, 1\right)$ we study the spectrum of the linearized Hamiltonian vector field $L_{h}=\omega_{N}^{-1}\left(\left.\mathbf{d}_{z}^{2} h_{\xi^{\perp}}\right|_{N}\right)$, where in our case $N$ and $\omega_{N}$ are as in Theorem 3.2. Also, from Proposition 6.2 in [21] we have

$$
\left.\left(\mathbf{d}_{F}^{2} h_{\xi^{\perp}}\right)\right|_{N}=\left[\begin{array}{ccc}
\operatorname{Ar} & 0 & 0 \\
0 & \left.\left(\mathbf{d}_{F}^{2} V_{\left(\xi_{L}, \xi_{R}\right)^{\perp}}^{\lambda}+\operatorname{corr}_{\left(\xi_{L}, \xi_{R}\right)^{\perp}}\right)\right|_{\mathbf{S}^{\mathrm{SL}(3)}} & 0 \\
0 & 0 & \ll \cdot, \cdot \gg_{\mathbf{S S L}^{\mathrm{SL}(3) *}}
\end{array}\right]
$$


Let us start by computing the block $\ll \cdot, \cdot \gg_{\mathrm{SSL}(3) *}$ of the above matrix, where $\ll$ $\cdot, \cdot \gg_{\mathbf{S}^{\mathrm{SL}(3) *} \text { is the inner product on the dual of } \mathbf{S}^{\mathrm{SL}(3)} \text { induced by the Riemannian }}$ metric on $\mathbf{S}^{\mathrm{SL}(3)}$. For, let as before fix the ordered basis $\left\{s_{1}, s_{2}, s_{3}\right\}$ on the slice $\mathbf{S}^{\mathrm{SL}(3)}$ where

$$
s_{1}=\left[\begin{array}{ccc}
1 & 0 & 0 \\
0 & 1 & 0 \\
0 & 0 & -2 \sqrt{1-e^{2}}
\end{array}\right] \quad s_{2}=\left[\begin{array}{ccc}
1 & 0 & 0 \\
0 & -1 & 0 \\
0 & 0 & 0
\end{array}\right] \quad s_{3}=\left[\begin{array}{lll}
0 & 1 & 0 \\
1 & 0 & 0 \\
0 & 0 & 0
\end{array}\right] .
$$

Recalling that $\ll A, B \gg_{\mathbf{S}^{\mathrm{SL}(3)}}=T \operatorname{tr}\left(A^{T} B\right)$ then the matrix that represents $\ll \cdot, \cdot \gg_{\mathbf{S S L}^{\mathrm{SL}}(3)}$ in the fixed basis is

$$
\mathcal{R}_{1}=2 T\left[\begin{array}{ccc}
3-2 e^{2} & 0 & 0 \\
0 & 1 & 0 \\
0 & 0 & 1
\end{array}\right]
$$

Let $\left\{s_{1}^{*}, s_{2}^{*}, s_{3}^{*}\right\}$ be the dual basis of $\left\{s_{1}, s_{2}, s_{3}\right\}$ under the identification of $\mathbf{S}^{\mathrm{SL}(3) *}$ with $\mathbf{S}^{\mathrm{SL}(3)}$ using the inner product $\ll \cdot, \cdot \gg_{\mathbf{S}^{\mathrm{SL}(3)}}$. In this basis the induced inner product $\ll \cdot, \cdot \gg_{\mathrm{SSL}^{\mathrm{S}(3) *}}$ is represented by $\mathcal{R}_{1}^{-1}$.

Let $\mathcal{R}_{2}=\left.\left(\mathbf{d}_{F}^{2} V_{\left(\xi_{L}, \xi_{R}\right)^{\perp}}^{\lambda}+\operatorname{corr}_{\left(\xi_{L}, \xi_{R}\right)^{\perp}}(F)\right)\right|_{\Sigma_{\text {int }}}$, then

$$
\left.\left(\mathbf{d}_{F}^{2} h_{\left(\xi_{L}, \xi_{R}\right)^{\perp}}\right)\right|_{N}=\left[\begin{array}{ccc}
A r & 0 & 0 \\
0 & \mathcal{R}_{2} & 0 \\
0 & 0 & \mathcal{R}_{1}^{-1}
\end{array}\right] .
$$

To compute $L_{h}$ in the basis $\left\{t_{1}, t_{2}, t_{3}, t_{4}, s_{1}, s_{2}, s_{3}, s_{1}^{*}, s_{2}^{*}, s_{3}^{*}\right\}$ for $N$ we use the formula for $\omega_{N}$ given in Theorem 3.2, Let us now compute each of the blocks of $\omega_{N}$.

Recall that $\mathfrak{q}^{\mu}=\mathfrak{t}$ for a MacLaurin spheroid. Then, from Theorem 3.2, for $\gamma_{1}, \gamma_{2} \in \mathfrak{t}$ we have:

$$
\Xi\left(\gamma_{1}, \gamma_{2}\right)=-\mu \cdot \operatorname{ad}_{\gamma_{1}} \gamma_{2}=-\left\langle\frac{2 \sqrt{2} T \Omega}{\left(1-e^{2}\right)^{1 / 3}} p, \operatorname{ad}_{\gamma_{1}} \gamma_{2}\right\rangle .
$$

Using (49), the matrix $\Xi$ is given by

$$
\Xi=\frac{2 T \Omega}{\left(1-e^{2}\right)^{1 / 3}}\left[\begin{array}{cccc}
0 & 0 & -1 & 0 \\
0 & 0 & 0 & 1 \\
1 & 0 & 0 & 0 \\
0 & -1 & 0 & 0
\end{array}\right]
$$

Since for a MacLaurin spheroid $\Sigma_{\text {int }}=\mathbf{S}^{\mathrm{SL}(3)}$ it is immediate from the definition of $\Psi$ in Theorem 3.2 that $\Psi$ is the zero matrix.

We now compute the Coriolis term $-\left.\mathbf{d} \chi^{\left(\xi_{L}, \xi_{R}\right)^{\perp}}\right|_{\Sigma_{\text {int }}}$. For that we will obtain a concrete expression for the right hand side of the equality

$$
\mathbf{d} \chi^{\left(\xi_{L}, \xi_{R}\right)^{\perp}}(X, Y)=X\left(\chi^{\left(\xi_{L}, \xi_{R}\right)^{\perp}}(Y)\right)-Y\left(\chi^{\left(\xi_{L}, \xi_{R}\right)^{\perp}}(X)\right)-\chi^{\left(\xi_{L}, \xi_{R}\right)^{\perp}}([X, Y]),
$$


with $X, Y \in \mathfrak{X}\left(\mathrm{GL}^{+}(3)\right)$.

Start by considering for $U, V \in T_{I} \mathrm{GL}^{+}(3)=\mathrm{L}(3)$ the corresponding leftinvariant extensions $X_{U}, X_{V} \in \mathfrak{X}\left(\mathrm{GL}^{+}(3)\right)$. We have $X_{U}(F)=F U$ for every $F \in \mathrm{GL}^{+}(3)$. Recall that $\left(\xi_{L}, \xi_{R}\right)^{\perp}=\frac{\Omega}{2}\left(\mathbf{e}_{\mathbf{3}},-\mathbf{e}_{\mathbf{3}}\right)$. Then according to the definition given in Theorem 3.2 we have

$$
\begin{aligned}
\chi^{\left(\xi_{L}, \xi_{R}\right)^{\perp}\left(X_{U}\right)(F)} & =\frac{\Omega T}{2} \operatorname{tr}\left(\left(\widehat{\mathbf{e}_{\mathbf{3}}} F+F \widehat{\mathbf{e}_{\mathbf{3}}}\right)^{T} F U\right) \\
& =-\frac{\Omega T}{2} \operatorname{tr}\left(\widehat{\mathbf{e}_{\mathbf{3}}}\left(F^{T} F U+F U F^{T}\right)\right) .
\end{aligned}
$$

It is straightforward to obtain

$$
\begin{aligned}
& X_{V}\left(\chi^{\left(\xi_{L}, \xi_{R}\right)^{\perp}}\left(X_{U}\right)\right)(F)= \\
& =-\frac{\Omega T}{2} \operatorname{tr}\left(\widehat{\mathbf{e}_{\mathbf{3}}}\left((F V)^{T} F U+F^{T} F V U+F V U F^{T}+F U(F V)^{T}\right)\right) \\
& =-\frac{\Omega T}{2} \operatorname{tr}\left(\widehat{\mathbf{e}_{\mathbf{3}}}\left(V^{T} F^{T} F U+F^{T} F V U+F V U F^{T}+F U V^{T} F^{T}\right)\right)
\end{aligned}
$$

Also, since $X_{U}, X_{V}$ are left-invariant vector fields, the identity $\left[X_{U}, X_{V}\right]=$ $X_{U V-V U}$ holds and we have, from (53)

$\chi^{\left(\xi_{L}, \xi_{R}\right)^{\perp}}\left(\left[X_{U}, X_{V}\right]\right)(F)=-\frac{\Omega T}{2} \operatorname{tr}\left(\widehat{\mathbf{e}_{\mathbf{3}}}\left(F^{T} F(U V-V U)+F(U V-V U) F^{T}\right)\right)$.

In order to compute $-\left.\mathbf{d} \chi^{\left(\xi_{L}, \xi_{R}\right)^{\perp}}\right|_{\Sigma_{\text {int }}}$ let $F=\operatorname{diag}(a, a, c)$ and $A, B \in \Sigma_{\text {int }}$. The unique left-invariant vector fields extending $A$ and $B$ are $X_{F^{-1} A}$ and $X_{F^{-1} B}$ respectively. Then, using (52) together with (54) and (55) it is immediate to obtain $-\left.\mathbf{d} \chi^{\left(\xi_{L}, \xi_{R}\right)^{\perp}}\right|_{\Sigma_{\text {int }}}=0$. Therefore, from Theorem 3.2 the symplectic matrix $\omega_{N}$ and its inverse are

$$
\omega_{N}=\left[\begin{array}{ccc}
\Xi & 0 & 0 \\
0 & 0 & \mathbf{1} \\
0 & -\mathbf{1} & 0
\end{array}\right] \quad \omega_{N}^{-1}=\left[\begin{array}{ccc}
\Xi^{-1} & 0 & 0 \\
0 & 0 & -\mathbf{1} \\
0 & \mathbf{1} & 0
\end{array}\right] .
$$

So the linearized vector field is

$$
L_{h}=\left[\begin{array}{ccc}
\Xi^{-1} \mathrm{Ar} & 0 & 0 \\
0 & 0 & -\mathcal{R}_{1}^{-1} \\
0 & \mathcal{R}_{2} & 0
\end{array}\right]
$$

where in our basis $\mathcal{R}_{2}=\operatorname{diag}\left(S_{1}, S_{2}, S_{2}\right)$ is given in (51).

The block $\Xi^{-1}$ Ar has imaginary eigenvalues $\epsilon_{1}^{ \pm}= \pm i \frac{\sqrt{8+e^{2}}}{2 e} \Omega$ with multiplicity 2. For the block

$$
\left[\begin{array}{cc}
0 & -\mathcal{R}_{1}^{-1} \\
\mathcal{R}_{2} & 0
\end{array}\right]
$$

we obtain the following eigenvalues:

- $\epsilon_{2}^{ \pm}= \pm i \sqrt{\frac{S_{1}}{\left(6-4 e^{2}\right) T}}$ with multiplicity 1 , and 
- $\epsilon_{3}^{ \pm}= \pm i \sqrt{\frac{S_{2}}{4 T}}$ with multiplicity 2 .

As $\left(6-4 e^{2}\right)$ and $S_{1}$ are positive for $0<e<1$, then $\epsilon_{2}^{ \pm}$is always imaginary in that range. However, $\epsilon_{3}^{ \pm}$becomes real if $S_{2}$ becomes negative, which happens exactly at $e_{0}$ as seen from the previous stability analysis. Hence, if $e>e_{0}$ the MacLaurin spheroid becomes linearly unstable, therefore unstable. This loss of stability corresponds exactly to a collision at 0 of $\epsilon_{3}^{ \pm}$for $e=e_{0}$, which passes from being pure imaginary to be real.

\section{Remark 6.1.}

1. In Riemann's work [19] some conclusions were made concerning the stability of Maclaurin spheroids by studying the existence of a minimum of a certain function $G$. The existence of this minimum was not done by studying the second variation of $G$. Riemann even says in page 188: "The direct analysis of the second variation of $G$ when the first variation vanishes would be very complicated; we can however decide if this function has a minimum by the following form:...". He follows with the analysis of the behavior of $G$. His final conclusion on the stability ends the paragraph 9 of his paper and is the following: "From this study it follows that the case of a rotation of an oblate ellipsoid, around its shortest axis, case already known to MacLaurin, can only be unstable if the relation between the shortest axis with the others is less than $0.303327 \ldots .$. . We note that if the relation between the shortest axis of the Maclaurin spheroid and the others is $\frac{c}{a}<0.303327$ this is equivalent to say that the eccentricity $e>e_{0}=0.952887$.

The value 0.303327 obtained by Riemann follows from his study on the existence of oblate spheroids in pages 184-185 of [19], namely as the root of the last displayed equation in page 184 . We remark that this equation is equivalent to the equation $S_{2}=0$ where $S_{2}$ is as in the proof of (i) in Theorem 6.2 Indeed

$$
S_{2}=0 \Longleftrightarrow e\left(1-e^{2}\right)\left(3+4 e^{2}\right)-\sqrt{1-e^{2}}\left(3+2 e^{2}-4 e^{4}\right) \arcsin e=0 .
$$

Taking $e=\cos \psi=\sin \left(\frac{\pi}{2}-\psi\right)$ and $\psi \in\left(0, \frac{\pi}{2}\right)$ one has

$$
\begin{aligned}
S_{2}= & 0 \Longleftrightarrow \\
& \cos \psi \sin ^{2} \psi(5+2 \cos (2 \psi))-\sin \psi\left(\frac{5}{2}-\cos (2 \psi)-\frac{1}{2} \cos (4 \psi)\right)\left(\frac{\pi}{2}-\psi\right)=0 \\
& \Longleftrightarrow 10 \sin (2 \psi)+2 \sin (4 \psi)+(-5+2 \cos (2 \psi)+\cos (4 \psi))(\pi-2 \psi)=0 .
\end{aligned}
$$

The equation (56) is the same one appearing in Riemann's paper. With respect to the computation of the root of this equation Riemann just says: "...this equation has, for $\psi$ between 0 and $\pi / 2$, the unique root $\sin \psi=0.303327 \ldots$..". Indeed, this is equivalent to say that $e_{0}=\cos \psi=0.952887 \ldots$

2. Concerning the stability of MacLaurin spheroids, it is shown in Liapunov's work [10] that under the hypothesis of the preservation of the ellipsoidal shape (setup we used) the value $e_{0}$ of loss of stability is the same as in Riemann's 
work, but if this hypothesis is dropped then the MacLaurin spheroid is only stable for $e<e_{1}$ with $e_{1}=0.8126 \ldots$. The point $e_{1}$ is exactly the point where the family of MacLaurin spheroids bifurcates into a branch of ellipsoids with 3 distinct axes lengths (Jacobi ellipsoids). We refer the reader to pages 52 and 61-63 of [10.

\subsection{Transversal spheroids}

All the qualitative properties, including stability, of two relative equilibria lying in the same orbit of the symmetry group are the same. Therefore, in view of Remark 5.1, in this subsection we analyze the nonlinear stability of the + family of transversal spheroids and the main result, Theorem 6.3. will follow for both families. We will set $\omega_{+}=\omega, f=f_{+}$and $\left(\xi_{L}, \xi_{R}\right)_{+}=\left(\xi_{L}, \xi_{R}\right)$ for notational simplicity. Also, to keep the notation consistent with the proof of Theorem 5.1 we will set $\mathbf{n}=\mathbf{e}_{\mathbf{2}}$.

In this case the computation of the splitting (10) is simplified due to the fact that $\mathfrak{g}_{p_{x}}=\{0\}$ and therefore $\mathfrak{p}=\mathfrak{g}_{\mu}$. Introducing the vectors $h=$

$\frac{1}{\sqrt{2}}\left(\mathbf{e}_{\mathbf{3}}, \mathbf{e}_{\mathbf{3}}\right), p_{1}=\left(\mathbf{e}_{2}, 0\right), p_{2}=\left(0, \mathbf{e}_{2}\right), t_{1}=\left(\mathbf{e}_{\mathbf{1}}, 0\right), t_{2}=\left(0, \mathbf{e}_{\mathbf{1}}\right), t_{3}=\frac{1}{\sqrt{2}}\left(\mathbf{e}_{\mathbf{3}},-\mathbf{e}_{\mathbf{3}}\right)$, we choose

$$
\begin{aligned}
\mathfrak{g}_{F} & =\operatorname{span}\{h\} \\
\mathfrak{p} & =\operatorname{span}\left\{p_{1}, p_{2}\right\} \\
\mathfrak{t} & =\operatorname{span}\left\{t_{1}, t_{2}, t_{3}\right\} .
\end{aligned}
$$

These subspaces are obviously invariant under the action of $G_{P_{F}}=\mathbb{Z}_{2}\left(\mathbf{e}_{2}\right)$. With respect to the basis $\left(p_{1}, p_{2}, t_{1}, t_{2}, t_{3}\right)$ for $\mathfrak{p} \oplus \mathfrak{t}$ we have,

$$
\widehat{\mathbb{I}}_{0}=T\left[\begin{array}{ccccc}
a^{2}+c^{2} & -2 a c & 0 & 0 & 0 \\
-2 a c & a^{2}+c^{2} & 0 & 0 & 0 \\
0 & 0 & a^{2}+c^{2} & -2 a c & 0 \\
0 & 0 & -2 a c & a^{2}+c^{2} & \\
0 & 0 & 0 & 0 & 4 a^{2}
\end{array}\right]
$$

Or in terms of the eccentricity of a prolate spheroid,

$$
\widehat{\mathbb{I}}_{0}=T\left[\begin{array}{ccccc}
\frac{2-e^{2}}{\left(1-e^{2}\right)^{2 / 3}} & \frac{-2}{\left(1-e^{2}\right)^{1 / 6}} & 0 & 0 & 0 \\
\frac{-2}{\left(1-e^{2}\right)^{1 / 6}} & \frac{2-e^{2}}{\left(1-e^{2}\right)^{2 / 3}} & 0 & 0 & 0 \\
0 & 0 & \frac{2-e^{2}}{\left(1-e^{2}\right)^{2 / 3}} & \frac{-2}{\left(1-e^{2}\right)^{1 / 6}} & 0 \\
0 & 0 & \frac{-e^{2}}{\left(1-e^{2}\right)^{1 / 6}} & \frac{2-e^{2}}{\left(1-e^{2}\right)^{2 / 3}} & 0 \\
0 & 0 & 0 & 0 & 4\left(1-e^{2}\right)^{1 / 3}
\end{array}\right] .
$$

It follows immediately that $\mathfrak{p}$ and $\mathfrak{t}$ are orthogonal with respect to $\widehat{\mathbb{I}}_{0}$, so our choice of the splitting $\mathfrak{g}=\mathfrak{g}_{F} \oplus \mathfrak{p} \oplus \mathfrak{t}$ is correct. In this basis the orthogonal velocity is

$$
\left(\xi_{L}, \xi_{R}\right)^{\perp}=\omega\left(p_{1}+f p_{2}\right),
$$

with $\omega$ and $f$ as in the + family in Theorem 5.1 .

It is straightforward to compute the adjoint representation of $\mathfrak{g}$ in this basis: 
Lemma 6.2. The elements of $\mathfrak{p} \oplus \mathfrak{t}$ satisfy the following relations:

$$
\begin{aligned}
& \operatorname{ad}_{t_{1}} t_{2}=0 \quad \operatorname{ad}_{t_{1}} t_{3}=-\frac{1}{\sqrt{2}} p_{1} \quad \operatorname{ad}_{t_{2}} t_{3}=\frac{1}{\sqrt{2}} p_{2} \\
& \operatorname{ad}_{t_{1}} p_{1}=\frac{1}{\sqrt{2}}\left(h+t_{3}\right) \quad \operatorname{ad}_{t_{1}} p_{2}=0 \\
& \operatorname{ad}_{t_{2}} p_{1}=0 \quad \operatorname{ad}_{t_{2}} p_{2}=\frac{1}{\sqrt{2}}\left(h-t_{3}\right) \\
& \operatorname{ad}_{t_{3}} p_{1}=-\frac{1}{\sqrt{2}} t_{1} \quad \operatorname{ad}_{t_{3}} p_{2}=\frac{1}{\sqrt{2}} t_{2} \\
& \operatorname{ad}_{p_{1}} p_{2}=0 .
\end{aligned}
$$

With this, we can prove the following proposition, which shows that the stability method is applicable.

Proposition 6.2. The Arnold form for a transversal spheroid is positive definite.

Proof. Recall that the momentum of a transversal spheroid is

$$
\mu=\widehat{\mathbb{I}}_{0}\left(\xi_{L}, \xi_{R}\right)^{\perp}=T \omega\left(\kappa^{L} p_{1}+\kappa^{R} p_{2}\right),
$$

where $\kappa^{L}:=\left[\left(a^{2}+c^{2}\right)-(2 / a) f\right]$ and $\kappa^{R}:=\left[\left(a^{2}+c^{2}\right) f-(2 / a)\right]$. From Lemma 6.2. and recalling that $\operatorname{ad}_{\gamma}^{*} \rho=-\operatorname{ad}_{\gamma} \rho$, we have, for $\gamma=\gamma^{(1)} t_{1}+\gamma^{(2)} t_{2}+\gamma^{(3)} t_{3} \in \mathfrak{t}$ :

$$
\mathbb{P}_{\mathfrak{g}_{F}}\left(\operatorname{ad}_{\gamma}^{*} \mu\right)=\frac{-T \omega}{\sqrt{2}}\left(\gamma^{(1)} \kappa^{L}+\gamma^{(2)} \kappa^{R}\right) h .
$$

This is zero iff $\gamma^{(2)}=-\kappa \gamma^{(1)}$, with

$$
\kappa:=\frac{\kappa^{L}}{\kappa^{R}}=-\frac{(e-1)(e+2)}{(e-2) \sqrt{1-e^{2}}},
$$

and therefore $\mathfrak{q}^{\mu}=\left\{\gamma^{(1)}\left(t_{1}-\kappa t_{2}\right)+\gamma^{(3)} t_{3}:\left(\gamma^{(1)}, \gamma^{(3)}\right) \in \mathbb{R}^{2}\right\}$. A basis for $\mathfrak{q}^{\mu}$ is given by $\left\{\gamma_{a}=t_{1}-\kappa t_{2}, \gamma_{b}=t_{3}\right\}$. Now, proceeding as for the MacLaurin spheroid, we compute

$$
\begin{gathered}
\operatorname{ad}_{\gamma_{a}}\left(\widehat{\mathbb{I}}_{0}^{-1} \mu\right)=\frac{\omega}{\sqrt{2}}\left((1-\kappa f) h+(1+\kappa f) t_{3}\right) \quad \operatorname{ad}_{\gamma_{b}}\left(\widehat{\mathbb{I}}_{0}^{-1} \mu\right)=\frac{\omega}{\sqrt{2}}\left(f t_{2}-t_{1}\right) \\
\operatorname{ad}_{\gamma_{a}}^{*} \mu=-\sqrt{2} T \omega \kappa^{L} t_{3} \quad \operatorname{ad}_{\gamma_{b}}^{*} \mu=\frac{T \omega}{\sqrt{2}}\left(\kappa^{L} t_{1}-\kappa^{R} t_{2}\right) \\
\widehat{\mathbb{I}}_{0}^{-1}\left(\operatorname{ad}_{\gamma_{a}}^{*} \mu\right)=\frac{-\kappa^{L} \omega}{2 \sqrt{2}\left(1-e^{2}\right)^{1 / 3}} t_{3} \\
\widehat{\mathbb{I}}_{0}^{-1}\left(\operatorname{ad}_{\gamma_{b}}^{*} \mu\right)=\frac{\left(1-e^{2}\right)^{1 / 6} \omega}{\sqrt{2} e^{4}}\left(\left(-\sqrt{1-e^{2}}\left(e^{2}-2\right) \kappa^{L}+2\left(e^{2}-1\right) \kappa^{R}\right) t_{1}\right. \\
\left.+\left(\sqrt{1-e^{2}}\left(e^{2}-2\right) \kappa^{R}-2\left(e^{2}-1\right) \kappa^{L}\right) t_{2}\right) .
\end{gathered}
$$


From where it easily follows that, in the basis $\left\{\gamma_{a}, \gamma_{b}\right\}$ for $\mathfrak{q}^{\mu}$,

$$
\operatorname{Ar}=\operatorname{diag}\left(\frac{3 e^{4}(2+e) T \omega^{2}}{2(2-e)\left(1-e^{2}\right)^{5 / 3}}, \frac{4(1+e)(2-e)(2+e) T \omega^{2}}{e^{2}\left(1-e^{2}\right)^{2 / 3}}\right) .
$$

The entries of Ar are obviously positive.

We can therefore apply the singular reduced energy-momentum method to study the stability of the transversal spheroid, obtaining the following

Theorem 6.3. Both families of transversal spheroids are nonlinearly stable for all eccentricities.

Proof. We start by computing the space of internal variations $\Sigma_{\text {int }}$. Recall that the slice $\mathbf{S}^{\mathrm{SL}(3)}$ at $F=\operatorname{diag}(a, a, c)$ is given by (50). Hence, for $\gamma=\left(\gamma^{(1)}, \gamma^{(3)}\right)$ in $\mathfrak{q}^{\mu}$ and $A=\left(a_{1}, a_{2}, a_{3}\right)$ in $\mathbf{S}^{\mathrm{SL}(3)}$ we have

$$
\gamma_{\mathrm{SL}(3)}(F)+A=\left[\begin{array}{ccc}
a_{1}+a_{2} & a_{3}-\sqrt{2} a \gamma^{(3)} & 0 \\
a_{3}+\sqrt{2} a \gamma^{(3)} & a_{1}-a_{2} & -(c+a \kappa) \gamma^{(1)} \\
0 & (a+c \kappa) \gamma^{(1)} & \frac{-2 c}{a} a_{1}
\end{array}\right] .
$$

Differentiating (24) we find

$$
\begin{array}{ll}
\left\langle h,\left(\mathbf{D I}(F) \cdot\left(\gamma_{\mathrm{SL}(3)}(F)+A\right)\right)\left(\xi_{L}, \xi_{R}\right)^{\perp}\right\rangle & =0 \\
\left\langle t_{1},\left(\mathbf{D I}(F) \cdot\left(\gamma_{\mathrm{SL}(3)}(F)+A\right)\right)\left(\xi_{L}, \xi_{R}\right)^{\perp}\right\rangle & =\frac{2(e+1) T \omega\left(e a_{3}+\sqrt{2}\left(1-e^{2}\right)^{1 / 6} \gamma^{(3)}\right)}{\left(1-e^{2}\right)^{5 / 6}{ }^{2}} \\
\left\langle t_{2},\left(\mathbf{D} \mathbb{I}(F) \cdot\left(\gamma_{\mathrm{SL}(3)}(F)+A\right)\right)\left(\xi_{L}, \xi_{R}\right)^{\perp}\right\rangle & =\frac{-2 T \omega\left(e a_{3}+\sqrt{2}\left(1-e^{2}\right)^{1 / 6} \gamma^{(3)}\right)}{\left(1-e^{2}\right)^{1 / 3}} \\
\left\langle t_{3},\left(\mathbf{D} \mathbb{I}(F) \cdot\left(\gamma_{\mathrm{SL}(3)}(F)+A\right)\right)\left(\xi_{L}, \xi_{R}\right)^{\perp}\right\rangle & =\frac{3 \sqrt{2} e^{3} T \omega \gamma^{(1)}}{(e-2)\left(1-e^{2}\right)^{2 / 3}} .
\end{array}
$$

In order for $\left(\mathbf{D I}(F) \cdot\left(\gamma_{\mathbf{S}^{\mathrm{SL}(3)}}+A\right)\right)\left(\xi_{L}, \xi_{R}\right)^{\perp} \in\left(\mathfrak{g}_{F} \oplus \mathfrak{t}\right)^{\circ}$ all the above expressions must vanish, which happens if and only if $\gamma^{(1)}=0$ and $\gamma^{(3)}=a_{3} \epsilon$, with $\epsilon=\frac{-e}{\sqrt{2}\left(1-e^{2}\right)^{1 / 6}}$. Therefore we can chose a basis for the space of internal variations $\Sigma_{\text {int }}$ such that any element $v$ belonging to it has components $\left(a_{1}, a_{2}, a_{3}\right)$ with the parametrization

$$
v=\left(a_{1}, a_{2}, a_{3}\right) \mapsto\left[\begin{array}{ccc}
a_{1}+a_{2} & a_{3}(1-\sqrt{2} a \epsilon) & 0 \\
a_{3}(1+\sqrt{2} a \epsilon) & a_{1}-a_{2} & 0 \\
0 & 0 & \frac{-2 c}{a} a_{1}
\end{array}\right] .
$$

It is straightforward to obtain

$$
(\mathbf{D I}(F) \cdot v)\left(\xi_{L}, \xi_{R}\right)^{\perp}=2 e T \omega\left(\frac{-\left((e-1) a_{1}+(e+1) a_{2}\right)}{\left(1-e^{2}\right)^{5 / 6}} p_{1}+\frac{(e+1) a_{1}+a_{2}(e-1)}{(e-1)\left(1-e^{2}\right)^{1 / 3}} p_{2}\right) .
$$

Then the correction term, for $v_{1}=\left(a_{1}, a_{2}, a_{3}\right), v_{2}=\left(b_{1}, b_{2}, b_{3}\right)$, is given by:

$$
\operatorname{corr}_{\left(\xi_{L}, \xi_{R}\right)^{\perp}}\left(v_{1}, v_{2}\right)=\frac{8 T \omega^{2}}{e-1}\left(\frac{9-5 e^{2}}{e^{2}-1} a_{1} b_{1}-3\left(a_{1} b_{2}+a_{2} b_{1}\right)-a_{2} b_{2}\right)
$$


For the restriction of the Hessian of $V_{\left(\xi_{L}, \xi_{R}\right)^{\perp}}^{\lambda}$ at $F$ we compute first the following second variations

$$
\begin{aligned}
& \mathbf{d}_{F}^{2} V\left(v_{1}, v_{2}\right)=D_{1} a_{1} b_{1}+D_{2} a_{2} b_{2}+D_{3} a_{3} b_{3}, \quad \text { with } \\
& D_{1}= \frac{4}{\left(1-e^{2}\right)^{5 / 3}}\left(-\left(1-e^{2}\right)^{2 / 3}\left(-3+e^{2}\right) V_{1}+3 e^{2}\left(e^{2}-1\right) V_{2}+4 e^{4} V_{11}\right. \\
&\left.+8 e^{4}\left(1-e^{2}\right)^{1 / 3} V_{12}+4 e^{4}\left(1-e^{2}\right)^{2 / 3} V_{22}\right) \\
& D_{2}= 4\left(V_{1}+\frac{e^{2}}{\left(1-e^{2}\right)^{2 / 3}} V_{2}\right) \\
& D_{3}= \frac{4}{e^{2}-1}\left(\left(e^{4}-1\right) V_{1}+e^{2}\left(1-e^{2}\right)^{1 / 3}\left(e^{2}-3\right) V_{2}\right) \\
&\left\langle\left(\xi_{L}, \xi_{R}\right)^{\perp},\left(\mathbf{D}_{F}^{2} \mathbb{I}\left(v_{1}, v_{2}\right)\right)\left(\xi_{L}, \xi_{R}\right)^{\perp}\right\rangle= \\
& 4 T \omega^{2}\left(\frac{\left(9-5 e^{2}\right)}{(e-1)^{2}(1+e)} a_{1} b_{1}+\frac{3}{1-e}\left(a_{1} b_{2}+a_{2} b_{1}\right)+\frac{1}{(1-e)} a_{2} b_{2}+(1+e) a_{3} b_{3}\right) \\
& \mathbf{d}_{F}^{2} \operatorname{det}\left(v_{1}, v_{2}\right)=\frac{-2}{\left(1-e^{2}\right)^{1 / 3}}\left(3 a_{1} b_{1}+a_{2} b_{2}+\left(1-e^{2}\right) a_{3} b_{3}\right)
\end{aligned}
$$

Putting all the contributions together, and substituting the integral expressions for the derivatives of the potential and the values of $\lambda$ and $\omega$ given in Theorem 5.1, we find that the matrix representing $\mathbf{d}_{F}^{2} V_{\left(\xi_{L}, \xi_{R}\right)^{\perp}}^{\lambda}+\left.\operatorname{corr}_{\left(\xi_{L}, \xi_{R}\right)^{\perp}}(F)\right|_{\Sigma_{\text {int }}}$ in the fixed basis of $\Sigma_{\text {int }}$ is block diagonal, with a $2 \times 2$ symmetric block in the first two components, and the other block given by the coefficient of $a_{3} b_{3}$, say $\phi$, which has the following expression:

$$
\phi=\frac{2}{\left(1-e^{2}\right)^{2 / 3}}\left[\left(1-e^{2}\right)^{2 / 3}\left(3+e^{2}\right) V_{1}+\left(3+2 e^{2}-e^{4}\right) V_{2}\right] .
$$

It is clear that $\phi$ is positive since $\left(3+2 e^{2}-e^{4}\right)$ is positive in $(0,1)$ and $V_{1}$ and $V_{2}$ are always positive.

The $2 \times 2$ block is $U=\frac{R}{e^{5}}\left[\begin{array}{ll}x & y \\ y & z\end{array}\right]$ with

$$
\begin{aligned}
& x=-27 e\left(1-e^{2}\right)+\left(27-36 e^{2}+17 e^{4}\right) \operatorname{arctanh} e \\
& y=-9\left(1-e^{2}\right)\left[3 e-\left(3-e^{2}\right) \operatorname{arctanh} e\right] \\
& z=-2 e\left(3-4 e^{2}\right)+2\left(3-5 e^{2}+2 e^{4}\right) \operatorname{arctanh} e
\end{aligned}
$$

The matrix $U$ has real eigenvalues. The trace and the determinant of $U$ are:

$$
\operatorname{tr}(U)=\frac{R}{e^{5}}\left[-e\left(33-35 e^{2}\right)+\left(33-46 e^{2}+21 e^{4}\right) \operatorname{arctanh} e\right]
$$


and

$$
\begin{aligned}
\operatorname{det}(U) & =\frac{R^{2}}{e^{10}}\left[-27 e^{2}\left(21-40 e^{2}+19 e^{4}\right)\right. \\
& -2\left(-567 e+1269 e^{3}-831 e^{5}+121 e^{7}\right) \operatorname{arctanh} e \\
& \left.-\left(567-1458 e^{2}+1212 e^{4}-334 e^{6}+13 e^{8}\right) \operatorname{arctanh}^{2} e\right] .
\end{aligned}
$$

These two quantities are positive in the interval $e \in(0,1)$. Their plots are displayed in Figure 2. Therefore, both families of transversal spheroids are stable for all eccentricities.
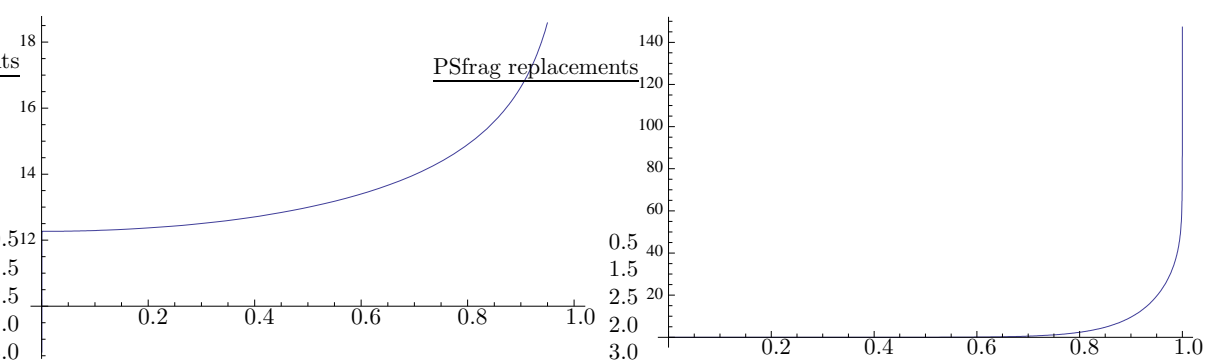

Figure 2: The plots of $\operatorname{tr}(U)$ (left) and $e^{10} \operatorname{det}(U)$ (right), in $R$ and $R^{2}$ units respectively.

\section{References}

[1] Arms, J.M., Marsden, J.E., and Moncrief, V. [1975], Symmetry and bifurcations of momentum mappings. Comm. Math. Phys, 78 455-478.

[2] Arnold, V.I. [1966], Sur la géometrie differentielle des groupes de Lie de dimension infinie et ses applications à l'hydrodynamique des fluids parfaits, Ann. Inst. Fourier, Grenoble, 16, 319-361.

[3] Chandrasekhar, S. [1987], Ellipsoidal figures of equilibrium, Dover Pub. Inc., New York.

[4] Ciarlet P.G. [1993], Mathematical Elasticity, volume 1: Three dimensional elasticity, North-Holland.

[5] Cohen, H., and Muncaster, R.G. [1988], The theory of pseudo-rigid bodies, New York: Springer-Verlag.

[6] Fassò, F. and Lewis, D. [2001], Stability properties of Riemann ellipsoids, Arch. Rational Mech. Anal. 158, 259-292. 
[7] Lerman, E. and Singer, S.F. [1998], Stability and persistence of relative equilibria at singular values of the moment map, Nonlinearity, 11, 16371649.

[8] Lewis, D. Marsden, J., and Ratiu, T.S. [1987], Stability and bifurcation of a rotating planar liquid drop, J. Math. Phys., 28, 10, 2508-2515.

[9] Lewis, D., Simo, J. C. [1990], Nonlinear stability of rotating pseudo-rigid bodies, Proc. Roy. Soc. London Ser. A, Vol. 427, 1873, 281-319.

[10] Liapounoff, M.A. [1904], Sur la stabilité des figures ellipsoidales d'équilibrie d'un liquide animé d'un movement de rotation, Annales de la Faculté des Sciences de L'Université de Toulouse.

[11] Marsden, J.E. [1992], Lectures on Mechanics, Lecture Note Series 174, LMS, Cambridge University Press.

[12] Marsden, J.E. and Hughes, T.J.R. [1983], Mathematical Foundations of Elasticity. Prentice-Hall.

[13] Montaldi J. [1997], Persistence and stability of relative equilibria, Nonlinearity, 10, 449-466.

[14] Ortega, J-P. and Ratiu, T.S. [1999], Stability of Hamiltonian relative equilibria, Nonlinearity, 12, 693-720.

[15] Patrick, G.W. [1992], Relative equilibria in Hamiltonian systems: the dynamics interpretation of nonlinear stability on the reduced phase space, $J$. Geom. Phys., 9, 111-119.

[16] Patrick, G.W., Roberts, M. and Wulff, C. [2004], Stability of Poisson and Hamiltonian relative equilibria by energy methods, Arch. Rational Mech. Anal., 174, 301-344.

[17] Perlmutter, M., Rodríguez-Olmos, M. and Sousa-Dias, M.E. The symplectic normal space of a cotangent-lifted action, Differential Geometry and Applications, to appear. Preprint math.SG/051207.

[18] Poincaré, H. [1885], Sur L'Equilibre d'une Masse Fluide Animée d'un Mouvement de Rotation, Acta Mathematica, 7, 259-380.

[19] Riemann, B. [1861], Ein Beitrag zu den Untersuchungen über die Bewegung cines flüssigen gleichartigen Ellipsoides, Abh. d. Königl. Gesell. der Wis. zu Göttingen, 9, 168-197.

[20] Roberts, M. and Sousa-Dias, M.E. [1999], Symmetries of Riemann ellipsoids, Resenhas- IME. USP, Vol. 4, 2, 183-221.

[21] Rodríguez-Olmos, M. [2006], Stability of relative equilibria in simple mechanical systems, Nonlinearity, 19, no.4, 853-877. 
[22] Rodríguez-Olmos, M. and Sousa-Dias, M.E. [2002], Symmetries of relative equilibria for simple mechanical systems. SPT 2002: Symmetry and perturbation theory (Cala Gonone), 221-230, World Sci. Publ.

[23] Rosensteel, G. [1988], Rapidly rotating nuclei as Riemann ellipsoids, Ann. Physics, 186, 230-291.

[24] Rosensteel, G. [1988], Geometric quantization of Riemann ellipsoids, Group theoretical methods in physics (Varna, 1987). Lecture Notes in Phys, 313, 253-260.

[25] Rosensteel, G. [2001], Gauge theory of Riemann ellipsoids, J. Phys. A, 34, 13, L169-L178.

[26] Simo J.C., Lewis, D. and Marsden J.E. [1991], Stability of relative equilibria. Part I: The reduced energy-momentum method, Arch. Rational Mech. Anal., 115, 15-59. 